\title{
THE SPITZER VIEW OF FR I RADIO GALAXIES: ON THE ORIGIN OF THE NUCLEAR MID-INFRARED CONTINUUM
}

\author{
C. Leipski ${ }^{1}$, R. Antonucci ${ }^{1}$, P. OGle ${ }^{2}$, And D. Whysong ${ }^{3}$ \\ ${ }^{1}$ Department of Physics, University of California, Santa Barbara, CA 93106, USA; leipski@ physics.ucsb.edu \\ ${ }^{2}$ Spitzer Science Center, California Institute of Technology, Mail Code 220-6, Pasadena, CA 91125, USA \\ ${ }^{3}$ National Radio Astronomy Observatory, P.O. Box O, Socorro, NM 87801, USA \\ Received 2008 October 6; accepted 2009 June 11; published 2009 July 28
}

\begin{abstract}
We present Spitzer mid-infrared (MIR) spectra of 25 FR I radio galaxies and investigate the nature of their MIR continuum emission. MIR spectra of star-forming galaxies and quiescent elliptical galaxies are used to identify host galaxy contributions while radio/optical core data are used to isolate the nuclear nonthermal emission. Out of the 15 sources with detected optical compact cores, four sources are dominated by emission related to the host galaxy. Another four sources show signs of warm, nuclear dust emission: 3C15, 3C84, $3 \mathrm{C} 270$, and NGC 6251. It is likely that these warm dust sources result from hidden active galactic nuclei of optical spectral type 1 . The MIR spectra of seven sources are dominated by synchrotron emission, with no significant component of nuclear dust emission. In parabolic spectral energy distribution fits of the nonthermal cores FR Is tend to have lower peak frequencies and stronger curvature than blazars. This is roughly consistent with the common picture in which the core emission in FR Is is less strongly beamed than in blazars.
\end{abstract}

Key words: galaxies: active - galaxies: jets - galaxies: nuclei

\section{INTRODUCTION}

Large double radio sources come in two radio-morphological types. The more powerful type is the "classical double" or FR II source, whose prototype is Cygnus A. This group has strong terminal "hotspots," and generally one-sided arcsecond (kpc) scale. The other type, FR Is like Centaurus A and M 87, is lower in radio luminosity, double-jetted, and edge-darkened (Fanaroff \& Riley 1974). These sources mostly have symmetric kiloparsec jets.

It has been known since the 1960s that some of these giant ( $\gtrsim 100 \mathrm{kpc}$ ) radio sources show powerful optical/UV continuum emission (called "big blue bump," or BBB) from a central point source. This optical/UV emission is generally identified as thermal radiation from dense matter accreting onto supermassive black holes. The gravitational potential energy of this infalling matter is thought to power much or all of the observed activity. Objects showing this strong optical/UV continuum and accompanying broad emission lines are classified as quasars and broad-line radio galaxies (hereafter, "quasars"). On the other hand, double radio sources without these components are called (narrow line) radio galaxies (NLRGs).

Many FR II NLRGs have hidden quasars detected by spectropolarimetry (e.g., Antonucci 1984; Ogle et al. 1997; Cohen et al. 1999) and recently also by their strong hot nuclear dust emission in the mid-infrared (MIR, e.g., Meisenheimer et al. 2001; Haas et al. 2004; Shi et al. 2005; Ogle et al. 2006; Cleary et al. 2007; Tadhunter et al. 2007). Thus, many powerful radio galaxies contain quasars which are hidden from direct view by dusty equatorial tori-i.e., as expected in the "unified model" (Barthel 1989; Antonucci 1993; Urry \& Padovani 1995).

The total light optical spectra of many FR II NLRGs already indicate the presence of a "classical" active galactic nucleus (AGN; those thought to accrete radiatively efficiently) by their high ionization emission-line ratios (high ionization galaxies
$\left.(\mathrm{HIGs})^{4}\right)$. Some FR II radio galaxies and most FR Is, however, show only weak line emission of low ionization and are therefore classified as low ionization galaxies (LIGs). Here, a luminous AGN (if present) has to be discovered at other wavelengths. Alternatively, these radio galaxies may simply lack a hidden quasar. A lack of a BBB means, according to current wisdom, a lack of a copious and radiatively efficient accretion flow. Historically such a situation was thought to require energy extraction from black hole rotation, and various mechanisms have been suggested for this (e.g., Begelman et al. 1984). Alternatively in principle accretion power could make the jet, yet not radiate significantly. In such a case the BBB is present, but of low power compared to a (lobe-matched) quasar BBB.

In an optical imaging study of 3CR FR I sources using Hubble Space Telescope (HST), Chiaberge et al. (1999) report unresolved central compact cores (CCCs) in the centers of most observed galaxies. The correlation of optical (at $\sim 7000 \AA$ ) compact cores and radio (at $6 \mathrm{~cm}$ ) cores in flux and luminosity is taken as evidence for their common origin as synchrotron emission from the base of a jet. Assuming that the base of the jet is found on scales smaller than any obscuring torus and considering the high detection rate of CCCs (85\%) they argue that we see directly into the nucleus and thus no standard, geometrically thick torus can be present in most low-luminosity (FR I) radio galaxies. Since no broad lines or strong BBB emission is observed there could also be no hidden, radiatively efficient AGN in those cases. However, e.g., Cao \& Rawlings (2004) note that if the emission from the jet occurs on scales larger than that of the torus the absence (or presence) of a torus cannot be directly inferred from the observed nuclear jet components. In addition, high-resolution $\mathrm{CO}$ maps have

\footnotetext{
4 Although such objects are usually referred to as high excitation galaxies (HEGs) the term HIGs seems more appropriate because the distinction is primarily one of ionization and not excitation, as expected for photoionized gases.
} 
revealed massive nuclear tori like those invoked in the standard unified model in the center of at least two FR I radio galaxies: 3C31 (Okuda et al. 2005) and NGC 3801 (Das et al. 2005). These tori have high molecular masses $\left(>10^{8} M_{\odot}\right)$, with high molecular column densities and probably geometrically thick shapes.

From an UV imaging study Zirbel \& Baum (2003) argue that the properties of FR Is are consistent with the classic unification models and the existence of an obscuring torus: while BL Lac objects are well aligned sources, FR I sources with nuclear UV components are at a critical angle for which a torus hides the nucleus but not the jet (or the latter greatly outshines the former). FR Is without nuclear UV components are thought to have a larger viewing angle where a torus hides the central regions completely.

Recently, some of the compact optical cores discovered by Chiaberge et al. (1999) have been studied with imaging polarimetry revealing fairly large polarization $(<11 \%)$ which further indicates their nonthermal origin, though such high polarizations can also result from scattering (Capetti et al. 2007).

In the X-rays, FR I sources are dominated by weakly absorbed nonthermal jet components in most cases (e.g., Balmaverde et al. 2006b; Evans et al. 2006). They lack the powerful absorbed, accretion-related component of a strong AGN (associated with a luminous accretion disk and circumnuclear obscuring structure). In many FR IIs, the latter is found in addition to the nonthermal jet component (Evans et al. 2006). Thus, in FR Is any nonjet accretion-related X-ray component carries much lower intrinsic AGN luminosities $\left(10^{39}-10^{41} \mathrm{erg} \mathrm{s}^{-1}\right)$ or else their detection is hampered by column densities of well above $10^{24} \mathrm{~cm}^{-2}$. Also Wu et al. (2007) argue that the (soft) X-rays in their FR I sample can be accounted for by jet emission only. We must note however that FR Is with broad emission lines in the optical do exist (e.g., 3C120; Tadhunter et al. 1993; see also Antonucci 2002a for an anecdotal listing of such objects). Such broad-line FR Is can extend up to quasar luminosities (Gower \& Hutchings 1984; Blundell \& Rawlings 2001; Heywood et al. 2007).

We here present a study of the MIR properties of a sample of FR I radio galaxies. The motivation is to possibly detect a hidden AGN by the reprocessed warm thermal dust emission as found in many FR II radio galaxies (e.g., Ogle et al. 2006). And even in the case of exceptionally high extinction which attenuates also the MIR luminosity, spectral features that give clues on the thermal or nonthermal origin of the emission might still be detectable. On the other hand, the nuclear part of the MIR spectra bridges the gap in wavelength between the optical compact cores and the radio cores observed by Chiaberge et al. (1999). If there is in fact no hidden AGN and no obscuring structure to be found in FR Is, the MIR spectra should agree with synchrotron core estimates.

\section{SAMPLE SELECTION AND OBSERVATIONS}

Our sample of FR I radio galaxies was selected from the 3CR (Spinrad et al. 1985) catalog (about half of the sources are also included in the $3 \mathrm{CRR}^{5}$ ). We selected a tractable subset of lobedominated sources with a flux limit of $\sim 15 \mathrm{Jy}$ at $178 \mathrm{MHz}$. To increase the sample size we added some very similar sources which were close to, but technically below, the flux cutoff. We also observed two additional sources because they were

\footnotetext{
5 http://3crr.extragalactic.info/
}

of special interest: $3 \mathrm{C} 189^{6}$ as well as IC 4296 . See Table 1 for some basic data of the selected sources. We note that our sample is not complete but can be considered representative for lobe-dominated FR I sources. No sources were lost by imposing scientifically relevant selections.

The morphological classification of the sources according to the Fanaroff-Riley scheme (Fanaroff \& Riley 1974) was checked using radio maps from the literature (see Table 1 for references).

The objects were observed in low-resolution mode with Infrared Spectrograph (IRS) (Houck et al. 2004) on board Spitzer Space Telescope (Werner et al. 2004), mostly under pid-20525. Each source under this pid was observed in staring mode for a total of $960 \mathrm{~s}, 1440 \mathrm{~s}, 720 \mathrm{~s}$, and $480 \mathrm{~s}$ in SL2, SL1, LL2, and LL1, respectively. After averaging all the cycles at one nod position we subtracted the off-order frames to remove the background. The resulting images were cleaned of residual rogue pixels and cosmic rays using Irsclean. The SPICE software was used to calibrate and extract one-dimensional spectra. The extracted spectra from the two nod positions were then averaged and data from the different modules were combined into a single spectrum. Due to the larger slit width in LL (10.7 in LL versus $3^{\prime \prime} .7$ in SL), multiplicative factors $(<1.5)$ were applied to the SL modules where necessary in order to match the flux levels.

Spectra for additional FR I sources not observed under pid-20525 (see Table 1, lower part) were also retrieved from the archive and were subject to the same procedures.

\section{THE SPITZER SPECTRA}

In Figures 1 and 2, we present the rest-frame MIR spectra for our FR I core sample and the supplemental objects. Because for 3C15 and 3C29 the SL modules of IRS suffer from saturated peak-up areas we present their spectra separately in Figure 3. Statements on the spectral behavior of the individual sources refer to the figures where their observed spectra are shown (Figures 1 and 2) and are thus considered in terms of $F_{v}$. All sources in our sample have been detected except for 3C403.1 which was not detected in either SL or LL.

\subsection{The Main Sample}

$3 C 31$ (NGC 383). This source shows very strong polycyclic aromatic hydrocarbon (PAH) emission and the MIR spectrum is likely to be dominated by emission related to star formation.

A massive molecular gas disk has been detected in $\mathrm{CO}$ in this object (Okuda et al. 2005).

$3 C 66 B$. This source shows increasing flux toward the shortest wavelengths observed (starting at around $8 \mu \mathrm{m}$ ) indicating a dominant contribution from the stellar component of the host galaxy at these wavelengths. A weak silicate feature in emission can be seen around $9.7 \mu \mathrm{m}$ as well as significant red continuum at longer wavelengths. Atomic line emission from low ionization species is detected but no PAH features.

$3 C 76.1$. While this source is clearly detected (as seen from the two-dimensional frames) it has low flux and shows a noisy spectrum. No spectral features can be securely identified. The spectrum shows slowly increasing flux toward longer wavelengths, starting at around $15 \mu \mathrm{m}$.

$3 C 83.1$ (NGC 1265). Emission from the host galaxy stellar population dominates this source up to $\sim 15 \mu \mathrm{m}$ where the

\footnotetext{
$63 \mathrm{C} 189$ is only part of the 3C catalog (Edge et al. 1959) but not of the 3CR or
} the 3CRR catalogs. See, e.g., Laing et al. (1983) for details on these samples. 
Table 1

The Sample

\begin{tabular}{|c|c|c|c|c|c|c|c|c|c|c|}
\hline Object & $\begin{array}{l}\text { Alternate } \\
\text { Name }\end{array}$ & $z$ & $\begin{array}{c}F_{178 \mathrm{MHz}} \\
(\mathrm{Jy})\end{array}$ & $\begin{array}{c}\text { Total } \\
\text { References }^{\mathrm{a}}\end{array}$ & $\begin{array}{c}F_{5 \mathrm{GHz}} \\
(\mathrm{mJy})\end{array}$ & $\begin{array}{c}\text { Core } \\
\text { References }^{\mathrm{a}}\end{array}$ & $\begin{array}{l}F_{\mathrm{CCC}}{ }^{\mathrm{b}} \\
(\mu \mathrm{Jy})\end{array}$ & $\begin{array}{l}\lambda_{\mathrm{CCC}} \\
\text { Filter }\end{array}$ & $\begin{array}{c}\text { Map } \\
\text { references }\end{array}$ & $\begin{array}{c}\text { Spectral } \\
\text { ref }^{\mathrm{a}}\end{array}$ \\
\hline $3 \mathrm{C} 15$ & & 0.073000 & 17.2 & 42 & 32 & 25 & 38 & F160W & 22 & 48 \\
\hline $3 \mathrm{C} 29$ & & 0.045031 & 16.5 & 42 & 93 & 24 & 10 & F702W & 24 & 48 \\
\hline $3 \mathrm{C} 31$ & NGC 383 & 0.017005 & 18.3 & 43 & 92 & 12 & 29 & F702W & 19 & 48 \\
\hline $3 \mathrm{C} 66 \mathrm{~B}$ & & 0.021258 & 26.8 & 43 & 182 & 12 & 97 & F702W & 14 & 48 \\
\hline $3 \mathrm{C} 76.1$ & & 0.032489 & 13.3 & 43 & 10 & 37 & $\ldots$ & $\ldots$ & 21 & 48 \\
\hline $3 \mathrm{C} 83.1$ & NGC 1265 & 0.025137 & 29.0 & 43 & 25 & 27 & 3 & F702W & 27 & 48 \\
\hline $3 \mathrm{C} 129$ & & 0.020800 & 51.1 & 44 & 34 & 38 & $\ldots$ & $\ldots$ & 36 & 48 \\
\hline $3 \mathrm{C} 189$ & NGC 2484 & 0.040828 & 7.4 & 45 & 195 & 13 & 76 & F814W & 7 & 1 \\
\hline $3 \mathrm{C} 264$ & NGC 3862 & 0.021718 & 28.3 & 43 & 200 & 12 & 248 & F791W & 41 & 28 \\
\hline $3 \mathrm{C} 270$ & NGC 4261 & 0.007465 & 56.5 & 42 & 308 & 24 & 11 & F791W & 9 & 33 \\
\hline $3 \mathrm{C} 272.1$ & M 84 & 0.003536 & 21.1 & 43 & 180 & 12 & 139 & F814W & 18 & 48 \\
\hline IC 4296 & & 0.012465 & 16.8 & 46 & 297 & 24 & 524 & F160W & 24 & 40 \\
\hline $3 C 293$ & & 0.045034 & 13.8 & 43 & 100 & 12 & $\ldots$ & $\ldots$ & 5 & 34 \\
\hline $3 \mathrm{C} 317$ & & 0.034457 & 53.4 & 42 & 391 & 24 & 23 & F814W & 6 & 48 \\
\hline NGC 6251 & & 0.024710 & 11.6 & 43 & 350 & 16 & 191 & $\mathrm{~F} 812 \mathrm{~W}$ & 30 & 23 \\
\hline $3 \mathrm{C} 386$ & & 0.016885 & 26.1 & 43 & 14 & 32 & 2301 & F702W & 21 & 48 \\
\hline $3 C 403.1$ & & 0.055400 & 14.7 & 42 & $\ldots$ & $\ldots$ & $\ldots$ & $\ldots$ & $\ldots$ & $\ldots$ \\
\hline $3 \mathrm{C} 424$ & & 0.126988 & 15.9 & 42 & 18 & 8 & $\ldots$ & $\ldots$ & 3 & 48 \\
\hline $3 \mathrm{C} 465$ & NGC 7720 & 0.030221 & 41.2 & 43 & 270 & 12 & 36 & F702W & 10 & 11 \\
\hline $3 \mathrm{C} 84$ & NGC 1275 & 0.017559 & 66.8 & 43 & 3100 & 26 & 3489 & F702W & 29 & 48 \\
\hline $3 \mathrm{C} 120$ & & 0.033010 & 7.4 & 45 & 1970 & 39 & $\ldots$ & $\ldots$ & 31 & 33 \\
\hline $3 \mathrm{C} 218$ & Hyd A & 0.054878 & 225.7 & 46 & 217 & 24 & $\ldots$ & $\ldots$ & 35 & 40 \\
\hline $3 \mathrm{C} 274$ & M 87 & 0.004360 & 1144.5 & 43 & 4000 & 12 & 894 & F814W & 15 & 48 \\
\hline BL Lac & & 0.068600 & $\sim 2.5^{\mathrm{c}}$ & 47 & $\sim 4.0^{\mathrm{c}}$ & $\ldots$ & $\ldots$ & $\ldots$ & 2 & 20 \\
\hline E1821+643 & & 0.297000 & $\sim 0.5$ & 47 & 8.0 & 4 & $\ldots$ & $\ldots$ & 4 & 17 \\
\hline
\end{tabular}

Notes.

${ }^{a}$ References for the total radio fluxes, radio core fluxes, radio maps, and optical spectra, respectively.

b Data taken from Chiaberge et al. (1999) except for 3C189 (Capetti et al. 2002), IC4296 (Balmaverde \& Capetti 2006a), and NGC 6251 (Chiaberge et al. 2003). Data on 3C15 provided by R. Baldi (2009, private communication).

${ }^{\mathrm{c}}$ The flux of only the extended emission is $29 \mathrm{mJy}$ at $20 \mathrm{~cm}$ (Antonucci 1986) which correspond to $\sim 151 \mathrm{mJy}$ at $178 \mathrm{MHz}$ (using $\left.\alpha=-0.8 ; S \propto v^{\alpha}\right)$. For the variable $5 \mathrm{GHz}$ core flux, we here give the average of all measurements available from NED.

References. (1) SDSS; (2) Antonucci 1986; (3) Black et al. 1992; (4) Blundell \& Rawlings 2001; (5) Bridle et al. 1981; (6) Burns 1990; (7) Capetti et al. 1993; (8) Chiaberge et al. 1999; (9) Condon \& Broderick 1988; (10) Condon et al. 1991; (11) De Robertis \& Yee 1990; (12) Giovannini et al. 1988; (13) Giovannini et al. 2005; (14) Hardcastle et al. 1996; (15) Hines et al. 1989; (16) Jones et al. 1986; (17) Kollatschny et al. 2006; (18) Laing \& Bridle 1987; (19) Laing et al. 2008; (20) Lawrence et al. 1996; (21) Leahy \& Perley 1991; (22) Leahy et al. 1997; (23) Miley \& Osterbrock 1979; (24) Morganti et al. 1993; (25) Morganti et al. 1999; (26) Taylor et al. 2006; (27) O'Dea \& Owen 1986; (28) Owen et al. 1996; (29) Pedlar et al. 1990; (30) Perley et al. 1984; (31) Simpson et al. 1996; (32) Strom et al. 1978; (33) Tadhunter et al. 1993; (34) Tadhunter et al. 2005; (35) Taylor et al. 1990; (36) Taylor et al. 2001; (37) Vallee 1982; (38) van Breugel \& Jägers 1982; (39) Walker et al. 1987; (40) Wills et al. 2004; (41) see http://www.jb.man.ac.uk/atlas/; (42) Kellermann et al. (1969), on Baars et al. (1977) scale using Laing \& Peacock (1980); (43) taken from http://3crr.extragalactic.info/; (44) estimated using GMRT measurements (Lal \& Rao 2004); (45) Kuehr et al. 1981; (46) estimated using the $160 \mathrm{MHz}$ flux (Kuehr et al. 1981) and $\alpha=-0.8$; (47) estimated from low-frequency measurements in NED; (48) Buttiglione et al. 2009.

spectrum starts to flatten in flux. PAH emission at $11.3 \mu \mathrm{m}$ and atomic emission from [Ne II] at $12.8 \mu \mathrm{m}$ are clearly detected. The ratio between the PAH features at $7.7 \mu \mathrm{m}$ and $11.3 \mu \mathrm{m}$ appears very small compared to, e.g., 3C31 (see Section 4.1).

3 C129. Here, the stellar emission again dominates below $\sim 10 \mu \mathrm{m}$ and for $\lambda>15 \mu \mathrm{m}$ strong MIR continuum emission is observed. We also detect weak silicate emission and PAHs at $11.3 \mu \mathrm{m}$.

$3 C 189$ (NGC 2484). This source appears very similar to 3C66B in the MIR spectrum with a distinct stellar component at short wavelengths, weak silicate emission around $9.7 \mu \mathrm{m}$, red continuum emission for $\lambda>10 \mu \mathrm{m}$, and no PAHs.

$3 C 264$ (NGC 3862). Strong PAH features are detected in this source which argues for a considerable, if not dominant, contribution from emission related to star formation. In fact, the steep continuum at $\lambda>20 \mu \mathrm{m}$ might well be associated with star formation. At the shortest wavelengths some minor contributions from the host galaxy stars can be identified.
$3 C 270$ (NGC 4261). A weak silicate feature and weak PAH emission is detected. While the blue spectral slope toward shorter wavelengths indicates contributions by stellar emission, a strong red continuum is observed for $\lambda>10 \mu \mathrm{m}$. In combination with the relatively weak PAH emission this continuum emission is not likely to originate from star-forming activity only.

3C272.1 (M 84-NGC 4374). A very unusual spectrum which has a blue slope up to $\sim 20 \mu \mathrm{m}$ and shows a very steep red slope at $\lambda>30 \mu \mathrm{m}$. Strong PAH emission is observed, but as in the case of $3 \mathrm{C} 83.1$ the $7.7 \mu \mathrm{m}-11.3 \mu \mathrm{m}$ ratio appears very small. Several atomic emission lines are detected, among them [O IV], a relatively high ionization line which can, however, also be found in star-forming galaxies (e.g., Smith et al. 2007).

IC 4296. Somewhat similar in appearance to 3C272.1 the continuum shows a red slope for $\lambda>20 \mu \mathrm{m}$ which steepens for $\lambda>30 \mu \mathrm{m}$. Weak PAH emission and strong atomic line emission are detected. The [O IV] emission line has much larger 

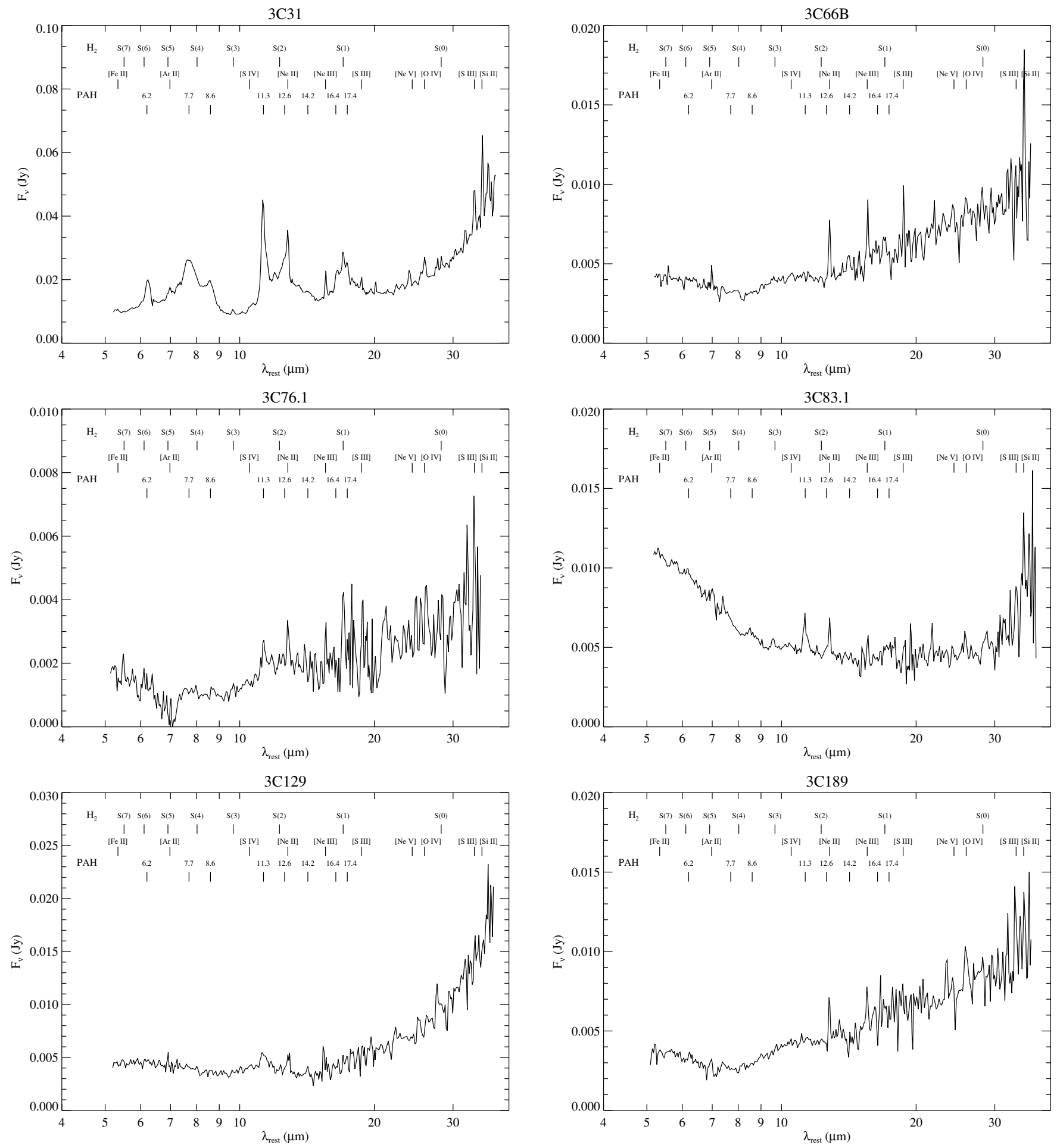

Figure 1. Observed MIR spectra of the FR I sources. Flux in Jy is plotted over the rest-frame wavelength in $\mu \mathrm{m}$.

equivalent width $(\mathrm{EW})$ than in $3 \mathrm{C} 272.1$ although the relative contributions from star formation to the spectrum, as traced by the PAHs, is apparently lower.

3C293. The MIR spectrum of this object is clearly dominated by star formation. The strong PAH features and the shape of the red continuum are very similar to what is seen in local star-forming galaxies (e.g., Smith et al. 2007). Notably, we also detect several rotational transitions from molecular hydrogen in emission (P. Ogle et al. 2009, in preparation).

$3 C 317$. This source shows a blue slope at the lowest wavelengths suggestive of stellar emission. Starting at around $10 \mu \mathrm{m}$ the spectrum turns to a red, but not very steep slope. We do not detect significant PAH features and [Ne II] $\lambda 11.3 \mu \mathrm{m}$ is the strongest atomic emission line. We do, however, detect faint emission from molecular hydrogen from $S(1)$ up to at least $S(6)$ (P. Ogle et al. 2009, in preparation).

$N G C$ 6251. Strong silicate emission at $9.7 \mu \mathrm{m}$ and $18 \mu \mathrm{m}$ is detected as previously noted in Ogle et al. (2007). Strong atomic and weak PAH emission can also be seen.

3C386. This source shows a very starlight dominated, blue continuum throughout the whole covered wavelength range. Some PAH features (e.g., $7.7 \mu \mathrm{m}$ and $11.3 \mu \mathrm{m}$ ) and atomic 

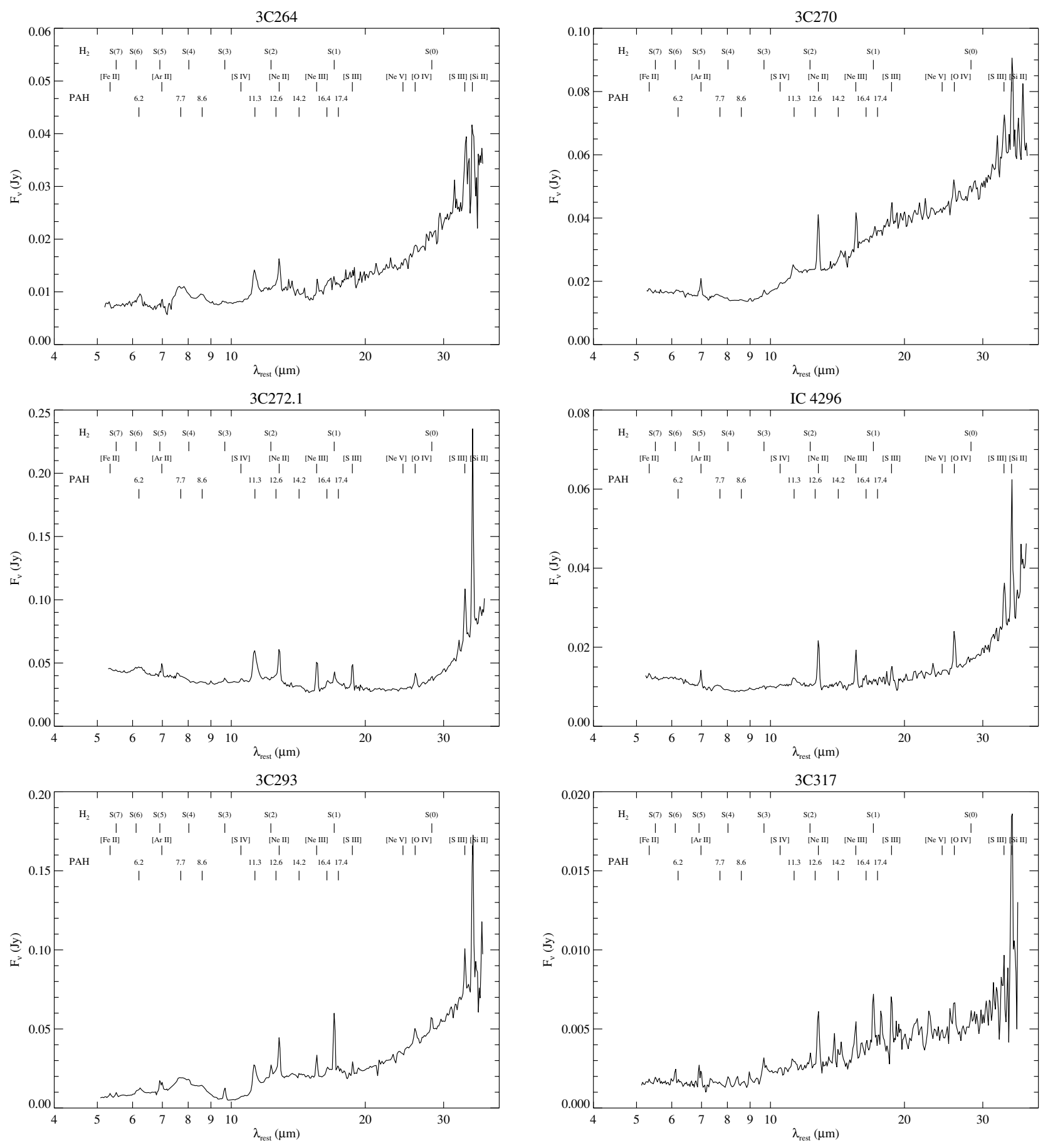

Figure 1. (Continued)

lines (e.g., [Ne II] $\lambda 12.8 \mu \mathrm{m}$ and [Ne III] $\lambda 15.6 \mu \mathrm{m}$ ) are present as well as weak molecular hydrogen emission. Simpson et al. (1996) tentatively detected a broad $\mathrm{H} \alpha$ line in the optical and Madrid et al. (2006) find that a foreground star falls on top of the nucleus for this galaxy (previously also noted by Lynds 1971).

3C424. The continuum emission is rather weak in this source but we detect it in all orders. The most notable feature in this spectrum is the exceptionally bright $\mathrm{S}(1)$ emission line from molecular hydrogen. Several other $\mathrm{H}_{2}$ emission lines can be identified as well.

$3 C 465$ (NGC 7720). While the presence of stellar emission can be identified at short wavelengths, the spectrum shows a prominent red slope for $\lambda>20 \mu \mathrm{m}$. Some atomic emission lines are detected on top of the rather noisy continuum and no significant PAH features (besides a possible detection of a weak $11.3 \mu \mathrm{m}$ feature) are found. Inspection of the two-dimensional spectral frames confirms that the source is detected in all orders, but especially in LL2 $(\sim 15-20 \mu \mathrm{m})$ it is of very low signal-tonoise ratio $(\mathrm{S} / \mathrm{N})$.

\subsection{The Supplemental Sample}

In Figure 2, we show the spectra of the FR I sources that do not belong to our initial sample. We use them here to demonstrate the diversity of MIR spectra seen for FR I sources. 

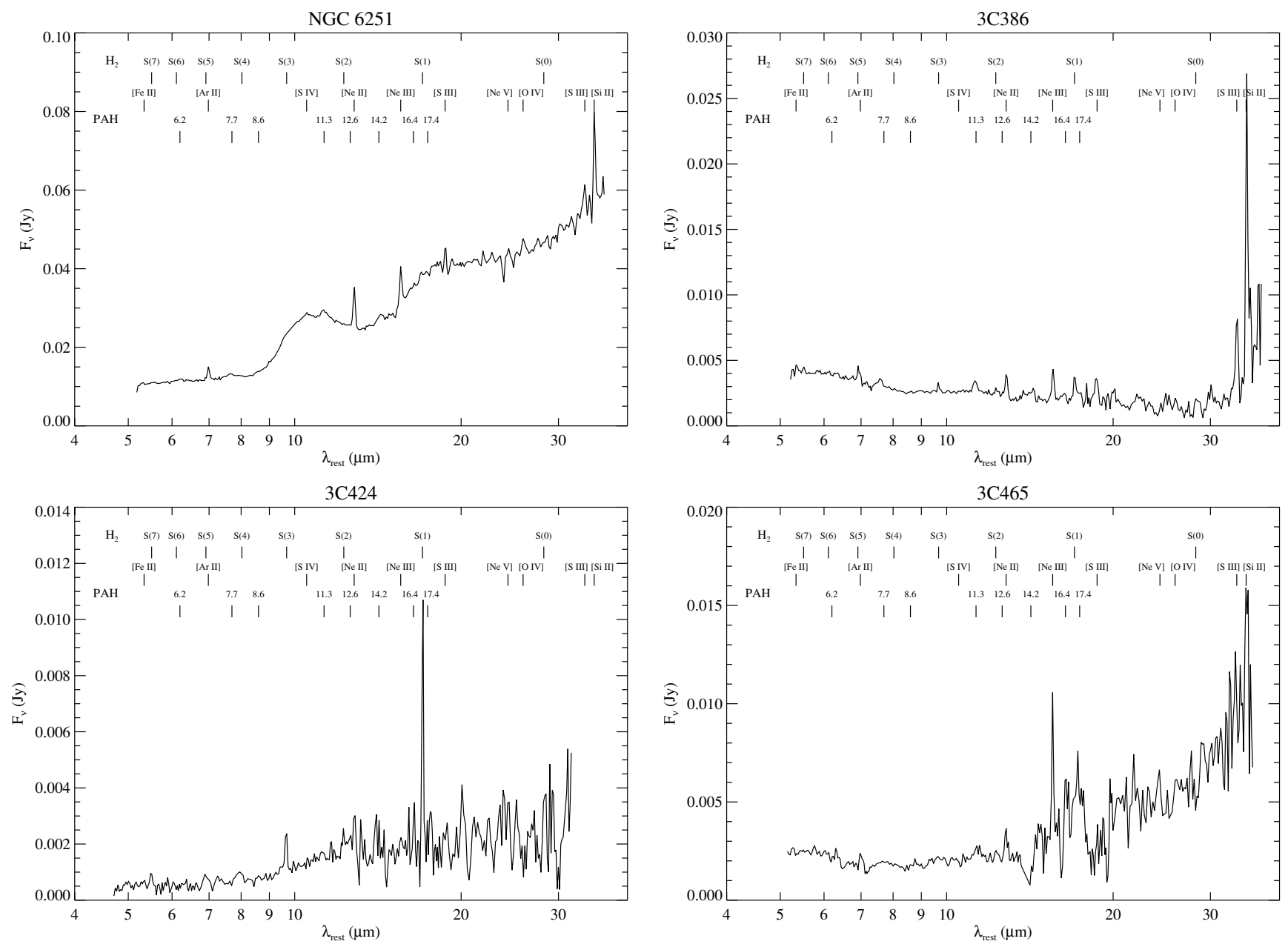

Figure 1. (Continued)

$3 C 84$ (Per A - NGC 1275). This source is highly core dominated in the radio (Pedlar et al. 1990). In the MIR, however, a strong thermal spectrum, including weak silicate emission and a steepening around $12 \mu \mathrm{m}$ is observed. It appears very similar in shape to the spectrum of the classical hidden AGN source 3C405 (=Cyg A; Figure 4) but the continuum appears much stronger in $3 \mathrm{C} 84$ relative to the emission lines.

3C120. This source is the only 3C FR I source in our sample where an AGN is already clearly visible at optical wavelengths as traced by broad emission lines and a blue optical/UV spectrum (Table 1). The Spitzer spectrum reflects this by showing silicate emission at $9.7 \mu \mathrm{m}$ and $18 \mu \mathrm{m}$, a rather flat continuum compared to sources like 3C84 and 3C405 and strong [Ne v] and [O IV] emission similar to the MIR spectra of typical type-1 AGN (e.g., Weedman et al. 2005; Buchanan et al. 2006).

$3 C 218$. Hydra A is the only source in our sample which is morphologically clearly a FR I but with a $178 \mathrm{MHz}$ luminosity that places it among the FR IIs. Optically a LIG (Table 1), the MIR spectrum shows PAH emission and [O IV] is also detected. Overall the spectrum looks quite similar to sources dominated by star formation in the MIR (e.g., 3C31, 3C293). However, here the continuum starts rising already at around 10-12 $\mu \mathrm{m}$ while for the other star-forming sources such a steepening in the continuum is observed around $\sim 20 \mu \mathrm{m}$ (Smith et al. 2007). Thus, it might have a warmer dust component or stronger nonthermal core contributions.
$3 C 274$ (M 87 - Vir A - NGC 4486). The MIR spectrum of M 87 shows a rather flat slope overlaid with some line emission from generally low ionization species (except for some weak [O IV] emission). At short wavelengths some contributions from the host galaxy can be identified and there is even some shallow silicate emission detected. For this source, high-resolution ground-based MIR flux measurements at $\sim 0$ '.5 resolution are also available (Perlman et al. 2001; Whysong \& Antonucci 2004).

BL Lac. As is common to sources in the class of objects named after this prototype, BL Lac is dominated by Dopplerboosted synchrotron emission at least from the radio through the optical. The MIR spectrum is also dominated by this synchrotron emission and shows a virtually featureless power-law continuum $(\alpha \sim-0.7)$. The diffuse radio emission suggests a relatively weak FR I source (Antonucci 1986). We show this object to illustrate a purely nonthermal spectrum.

$E 1821+643$. This source was first classified as a radio-quiet quasi-stellar object (QSO), but discovered in a survey at low radio frequencies (Lacy et al. 1992). Deep radio imaging, however, revealed a $\sim 300 \mathrm{kpc}$ FR I radio structure associated with this source (Blundell \& Rawlings 2001). Being a broad-line object the MIR spectrum looks quite similar to those of most other quasars or broad-line objects (e.g., 3C120). We clearly detect silicate emission at $9.7 \mu \mathrm{m}$ and a weak emission feature at $18 \mu \mathrm{m}$. We also detect [Ne v] emission and weak PAH emission (e.g., at $11.3 \mu \mathrm{m}$ ). The continuum longward of $\sim 10 \mu \mathrm{m}$ is redder 

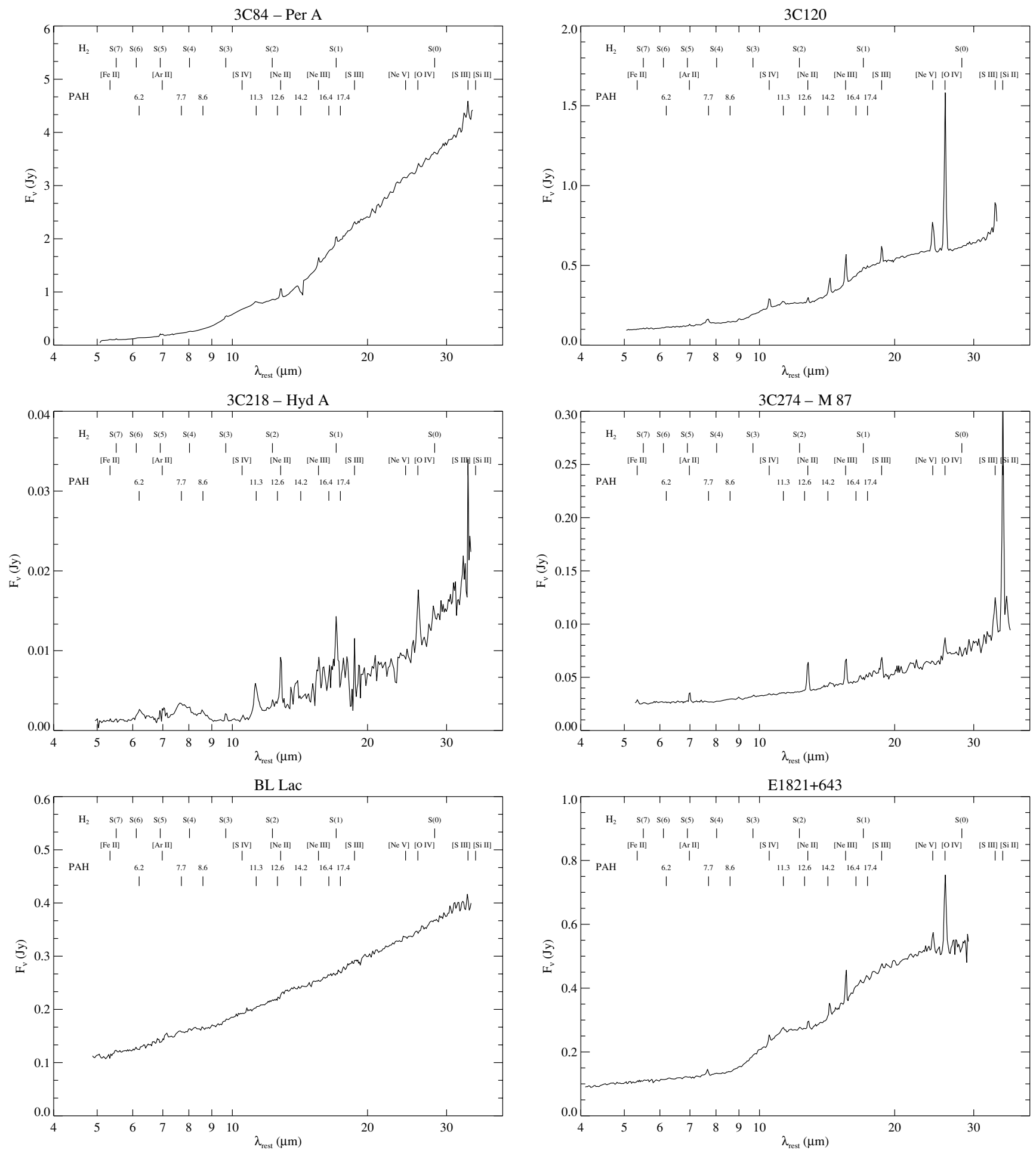

Figure 2. Observed MIR spectra of the supplemental objects. Again we plot the flux in Jy over the rest-frame wavelength in $\mu$ m.

than for 3C120 but not as red as for hidden AGN sources like Cyg A.

In Figure 3, we present the MIR spectra of $3 \mathrm{C} 15$ and 3C29. The spectra of these objects suffer from "spillover" due to saturated peak-up areas. Therefore, the flux levels and the slopes in the SL modules are likely to be compromised. It is notable, however, that both sources clearly show a red continuum at wavelengths greater than $15 \mu \mathrm{m}$ but no significant PAH emission. Since the red continuum is detected in the LL orders it is not an artifact of the spillover.

\section{MIR EMISSION IN FR I RADIO GALAXIES}

In this paper, we study the nuclear MIR emission of FR I radio galaxies and try to determine its properties and possible origins. Components considered are: (1) synchrotron emission from a nonthermal core; (2) warm dust emission resulting from AGN heating; (3) dust heated by star formation as traced by PAH features; and (4) stellar emission (photospheric emission plus dust emission associated with the circumstellar envelopes of AGB stars). We often summarize contributions 3 and 4 as processes from the host galaxy. We use the term 
$3 \mathrm{C} 15$

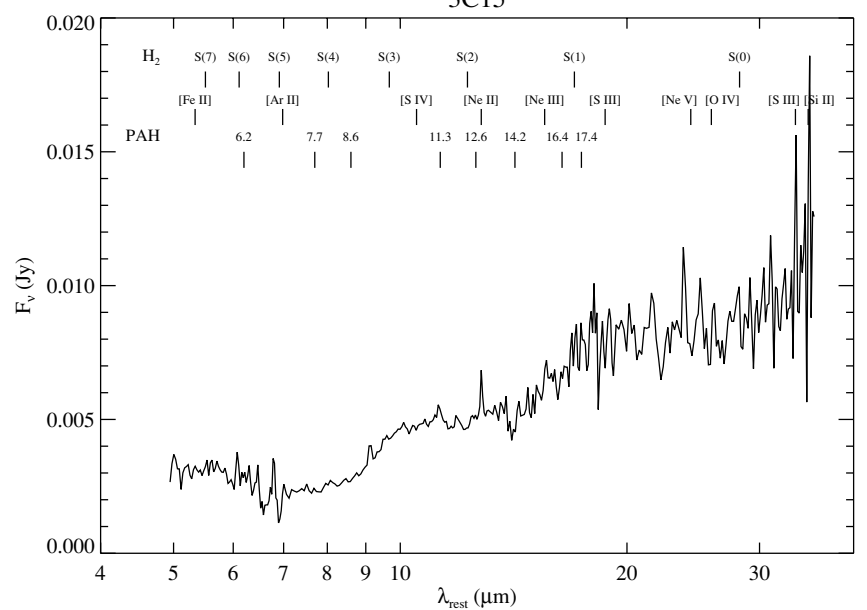

$3 \mathrm{C} 29$

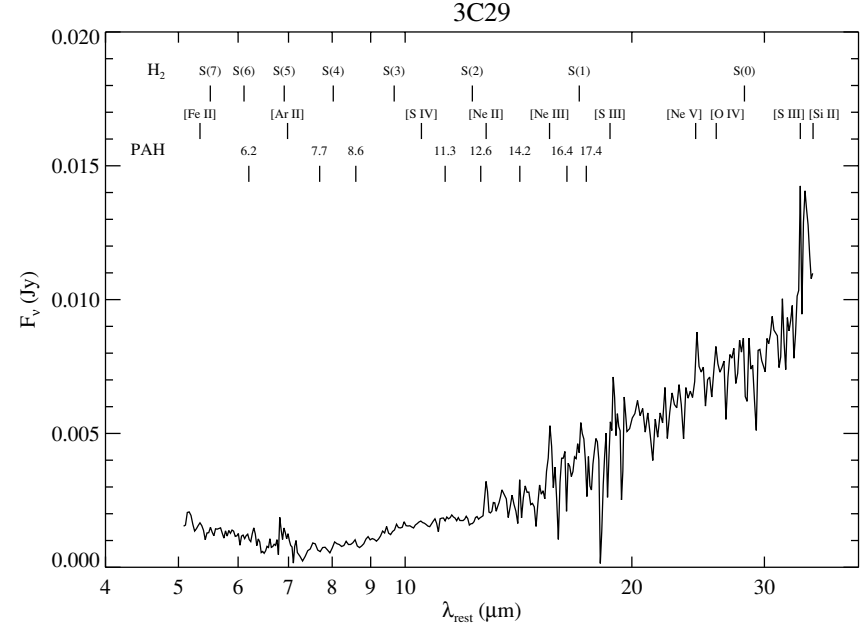

Figure 3. MIR spectra of 3C15 (left) and 3C29 (right).

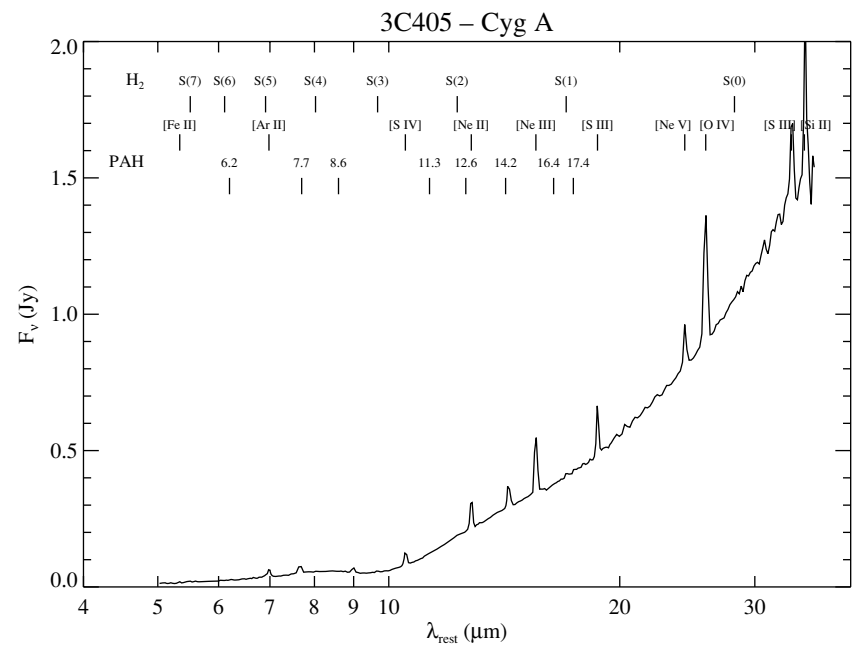

Figure 4. Observed MIR spectrum of 3C405, a prototypical hidden AGN source.

"nuclear" to address central emission related directly to an AGN."

In the framework of models which favor the absence of nuclear dust with high covering factors in FR I sources, the MIR spectra should consist of a combination of nonthermal emission due to a nuclear synchrotron source and more extended emission related to processes in the host galaxy. The warm/hot dust component related to an AGN should be missing or of very low luminosity. In order to explore any nuclear MIR emission in FR I sources, we will here test this scenario by successively identifying contributions from different mechanisms to the MIR spectra.

\subsection{Contributions from Star Formation}

Due to the large slit width of IRS (3".7 and 10'.7 for SL and LL, respectively) contributions from the host galaxy (star formation and stellar emission) to the MIR continuum can be significant and even dominant. We need to correct our spectra for these host galaxy contributions in order to explore any additional nuclear MIR emission.

\footnotetext{
7 We note that the definite test for the nuclear origin of the detected MIR emission would be high spatial resolution MIR imaging in order to compare the fluxes of any subarcsec nuclear point sources with the Spitzer fluxes (see, e.g., Whysong \& Antonucci 2004 for a discussion of the contrasting results on M 87 and Cen A)
}

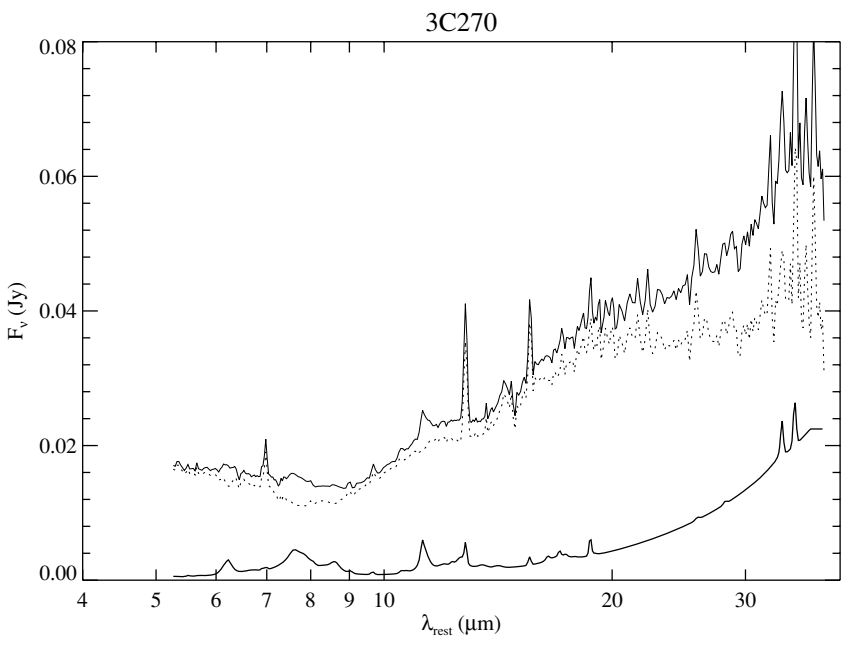

Figure 5. 3C270 as an example for the subtraction of the star-forming template (thin solid line) which is scaled to remove the $11.3 \mu \mathrm{m}$ PAH feature. The spectrum on the top is the total observed spectrum while the dotted line shows the star formation corrected spectrum (note the absence of the $11.3 \mu \mathrm{m} \mathrm{PAH}$ feature).

Because emission related to star formation has intrinsically a red spectral slope it cannot be distinguished easily from the synchrotron core emission or AGN-heated dust. Therefore, in order to securely identify any synchrotron or AGN dust components in the observed MIR spectra, emission from star formation needs to be corrected for. As an indicator for starforming contributions we here use the PAH features which are commonly observed in the spectra of star-forming galaxies and are a well known tracer of star formation activity. Only if PAHs are detected do we correct the spectra for emission related to star formation. No significant PAH emission usually indicates negligible continuum contribution from star formation compared to other processes.

In the case of detected PAH emission, we chose to subtract appropriately scaled average star formation template spectra taken from Smith et al. (2007). We used the template with the reddest MIR/FIR slope which was still in accordance with the data after scaling, thus being conservative about any residual emission. The template was scaled to the $11.3 \mu \mathrm{m}$ PAH feature which is strong, largely free of contaminations by atomic emission lines, and close to wavelengths where AGN-related emission usually has a large contribution. The star-forming 

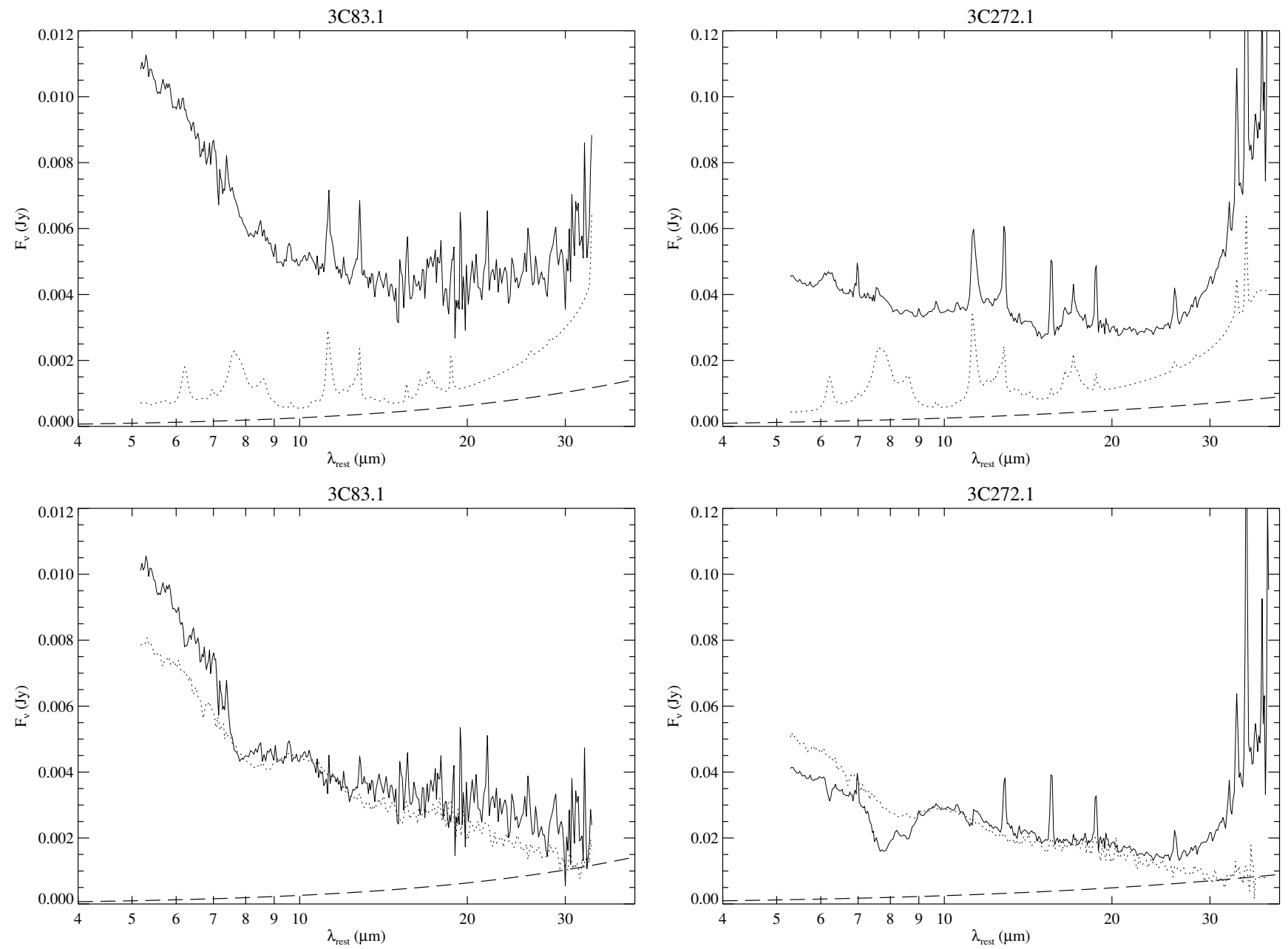

Figure 6. Unusual PAH ratios in 3C83.1 (left) and 3C272.1 (right). The top panel shows the observed spectrum with the star formation template scaled to the $11.3 \mu \mathrm{m}$ PAH feature. The bottom panel shows the results of the subtraction and the scaled spectrum of an early-type galaxy (NGC 1549). Approximate contributions from the synchrotron core (Section 4.4) are shown as long-dashed lines.

template was scaled in such a way that, after subtraction, the $11.3 \mu \mathrm{m}$ PAH feature is removed from our FR I spectrum (see Figure 5 for illustration). In Tables 2 and 3, we give estimates for the percentage contributions from star formation to the observed MIR flux at $15 \mu \mathrm{m}$ and $30 \mu \mathrm{m}$.

While this strategy works well for many of our sources, subtraction of the star formation template (scaled to the $11.3 \mu \mathrm{m}$ PAH feature) leaves a clear depression at the location of the $7.7 \mu \mathrm{m}$ feature for $3 \mathrm{C} 83.1$ and $3 \mathrm{C} 272.1$. This cannot be explained by the shape of stellar emission and argues for a very low $7.7 \mu \mathrm{m} / 11.3 \mu \mathrm{m}$ PAH ratio in these FR I galaxies (Figure 6). Apart from the low PAH ratio both spectra can be explained by a combination of processes in the host galaxy. We detect no significant nuclear continuum (but see Section 4.3).

Such low PAH ratios have already been noted in local (earlytype) galaxies (Kaneda et al. 2005, 2008; Smith et al. 2007). Sources with MIR continua dominated by star formation usually show no large variation in the $7.7 \mu \mathrm{m}-11.3 \mu \mathrm{m}$ ratio (Brandl et al. 2006; Smith et al. 2007) and similar star-formationdominated PAH ratios have been found in luminous radioquiet and radio-loud AGN (Shi et al. 2007b). In the absence of strong contributions from star formation, spectra with unusual $7.7 \mu \mathrm{m} / 11.3 \mu \mathrm{m}$ values can be observed. Interestingly, most sources with such low PAH ratios show low-luminosity AGN activity (Smith et al. 2007). Consistent with this, Sturm et al. (2006) have shown that IR-faint LINERs (LINERs without much star formation) show these unusual PAH ratios, while IR-luminous $\left(L_{\mathrm{IR}} / L_{B} \gtrsim 1\right.$ with $L_{\mathrm{IR}}$ being the $8-1000 \mu \mathrm{m}$ luminosity) LINERs have PAH ratios similar to starburst galaxies. In the former cases, it appears that the nuclear source powers most of the measured PAH emission. The harder spectrum could potentially lead to the destruction of the carriers of the $7.7 \mu \mathrm{m}$ band or to the preferential excitation of the $11.3 \mu \mathrm{m}$ PAH, thus explaining the observed relative decrease in the $7.7 \mu \mathrm{m}$ feature (Smith et al. 2007; Kaneda et al. 2008, and references therein). This could in principle indicate that the PAHs in 3C83.1 and 3C272.1 are not excited by star formation but by (lowluminosity) AGN activity instead. Any corrections made to the spectrum by the subtraction of a star-forming template would thus remove MIR continuum emission which is in fact powered by the AGN.

\subsection{Contributions from Stellar Emission}

Recall that we have already seen in the spectra (Figure 1) that contributions from stellar emission of the host galaxy can be substantial, particularly at shorter wavelengths. Most or all of our FR I sources reside in elliptical galaxies (e.g., Govoni et al. 2000; Madrid et al. 2006). The MIR spectra of early-type galaxies generally show blue colors throughout 

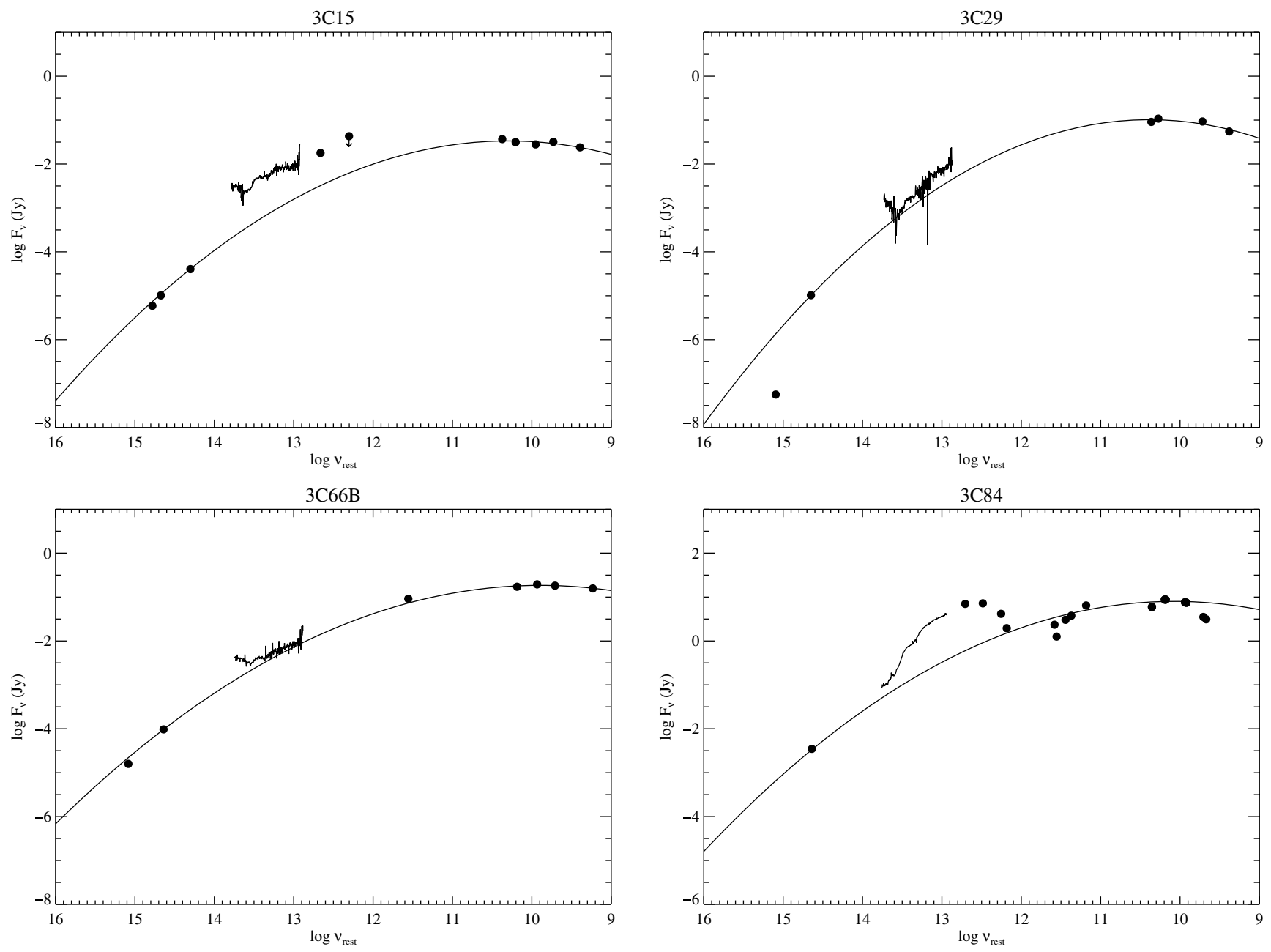

Figure 7. Observed nuclear SEDs for sources in which significant residual continuum emission could be identified. We used optical fluxes (Table 1), corrected for galactic absorption, nuclear NIR fluxes, and radio core flux measurements (arcsecond and subarcsecond scales). Only optical/NIR and radio core measurements were used for the parabolic fits. Nuclear UV (Chiaberge et al. 2002) fluxes, also corrected for galactic absorption, MIR/FIR, and submillimeter data (if available) as well as MIR spectra (this work) are overplotted. For sources with significant PAH features, we have subtracted the star formation component (3C84, 3C270, IC4296, NGC 6251; see the text).

the IRS wavelength range(e.g., Bressan et al. 2006; Kaneda et al. 2008) and even if moderate amounts of residual star formation are observed, the blue spectral slopes of the stellar emission can remain dominant in the total spectrum (Kaneda et al. 2008). This is supported by the fact that the spectral energy distributions (SEDs) of typical early-type galaxies without signs for considerable star formation usually show a blue IR slope that, due to emission from cold dust, turns over into a red slope at wavelengths beyond the IRS coverage (e.g., Dale et al. 2005; Kaneda et al. 2007; Temi et al. 2007). Therefore, the stellar contributions in our MIR spectra will have opposite spectral slope compared to any underlying synchrotron core or warm dust emission.

As shown by, e.g., Bressan et al. (2006) the MIR spectra of nonactive early-type galaxies can be well explained by including the dusty atmospheres and envelopes of AGB stars into stellar evolution models. The emission from dust produced in the atmospheres of AGB stars accounts for excess continuum emission over the long-wavelength quasi-Rayleigh-Jeans extrapolation of late-type stars as well as for the presence of silicate emission in the spectra of early-type galaxies.

We estimate contributions from the stellar population by using the observed spectrum of a quiescent elliptical galaxy which is scaled to the blue part of the spectra $(\sim 5-8 \mu \mathrm{m})$. For a template we chose the quiescent early-type galaxy NGC 1549 which is fairly bright at MIR wavelengths, does not show any PAH or atomic line emission, and has full IRS low-resolution coverage (Kaneda et al. 2008). We obtained the data for NGC 1549 from the Spitzer archive and extracted a spectrum following the procedures described in Kaneda et al. (2008). In Tables 2 and 3, we list estimated percentage contributions of the stellar component to the observed MIR flux at two wavelengths.

\subsection{Spectral Components in the MIR}

After the subtraction of the star-forming template in the way described above and after considering the stellar host galaxy emission we recognize basically two types of spectra.

1. Sources where the observed MIR emission can be accounted for by processes in the host galaxy.

The spectra are either dominated by star formation (3C31, 3C218, 3C264, 3C293) or the observed MIR emission can be explained by a combination of stellar and star-forming contributions (i.e., after the subtraction of the star-forming template the residual spectrum is largely consistent with stellar emission from an early-type galaxy; 3C76.1, 3C386; Figure 13 in the Appendix). 

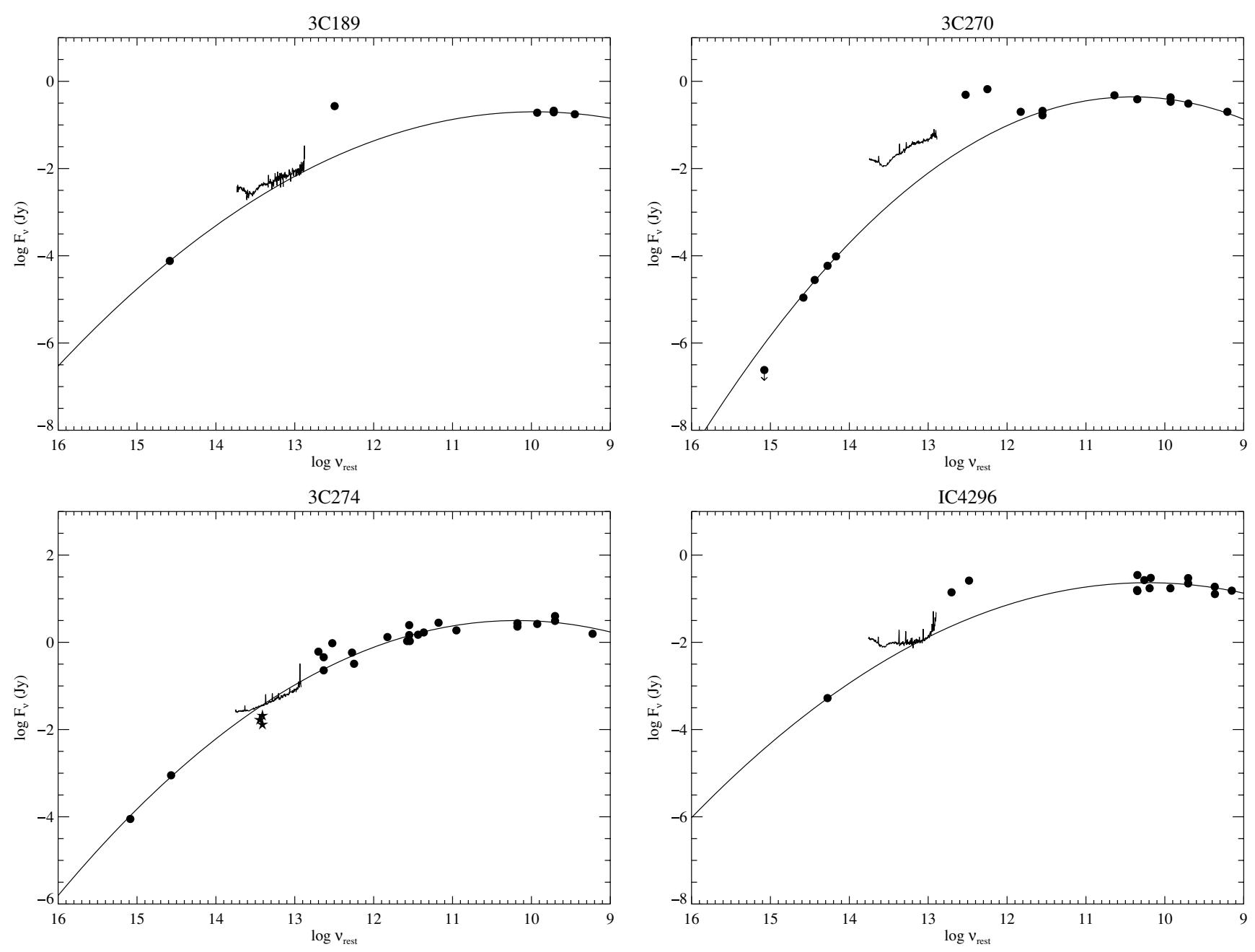

Figure 7. (Continued)

2. Objects which show significant residual emission at wavelengths longer than $\sim 8 \mu \mathrm{m}$ with a red spectral slope.

Because even star formation and stellar emission combined cannot explain these residual continua they must originate from other processes, for example warm (few hundred K) dust emission or nonthermal synchrotron emission. In some of the sources where residual continuum emission at $\lambda>8 \mu \mathrm{m}$ could be identified we also detect PAHs (3C84, 3C120, 3C129, 3C270, IC4296, NGC 6251, E1821+643; Figures 13 and 14 in the Appendix), but the EWs of these features are generally small. For many sources only red continuum emission at $\lambda>8 \mu \mathrm{m}$ (plus possibly stellar host galaxy emission) was detected (3C15, 3C29, 3C66B, 3C189, 3C274, 3C317, 3C424, 3C465, BL Lac). No significant PAH emission can be seen in their spectra.

As mentioned above in the case of 3C83.1 and 3C272.1 we cannot without doubt attribute the PAH emission to star formation. We count these objects as host-dominated sources, an interpretation which is supported by the shape of their MIR continuum (Figure 6), but we emphasize that we cannot be sure at this point if the PAH emission is excited by star formation or (low-luminosity) AGN activity.

\subsection{Comparison with Synchrotron Prediction}

Since it has been proposed that nonthermal emission might be a major, if not dominant constituent of the MIR emission in FR I sources, we also analyze the MIR spectra in the framework of their (nuclear) SEDs. As a comparison for the synchrotron core emission we chose to use well observed blazars and related objects because they represent nearly pure synchrotron emission.

It has been shown that, globally over a large frequency interval, the strongly beamed core emission of blazars can be well approximated by a parabolic function in $\log F_{v}$ versus $\log v$ (e.g., Landau et al. 1986). Following these authors, we here utilize a function of the form

$$
\log F_{v}=C+(\log v-B)^{2} / 2 A
$$

to represent the underlying beamed emission from the base of the jet ( $C$ is the $\log$ of the peak flux, $B$ is the log of the peak frequency of the parabola, and $A$ represents a "curvature" parameter). We note that a similar parabolic function can also be used to described the SEDs of BL Lacs in $\log v F_{v}$ versus $\log v$ (e.g., Nieppola et al. 2006).

In order to construct the (nuclear) SEDs, photometric data from the literature have been compiled from optical through radio wavelengths. For the radio and optical we only focused on core emission and we only compiled these data for sources with detected optical CCC components. In total, our sample has 14 objects in common with the sample of Chiaberge et al. (1999). But for two sources no CCCs could be identified: for 3C293 Chiaberge et al. (1999) could not estimate any CCC flux due to large-scale dust structures in the host galaxy, and $3 \mathrm{C} 424$ 

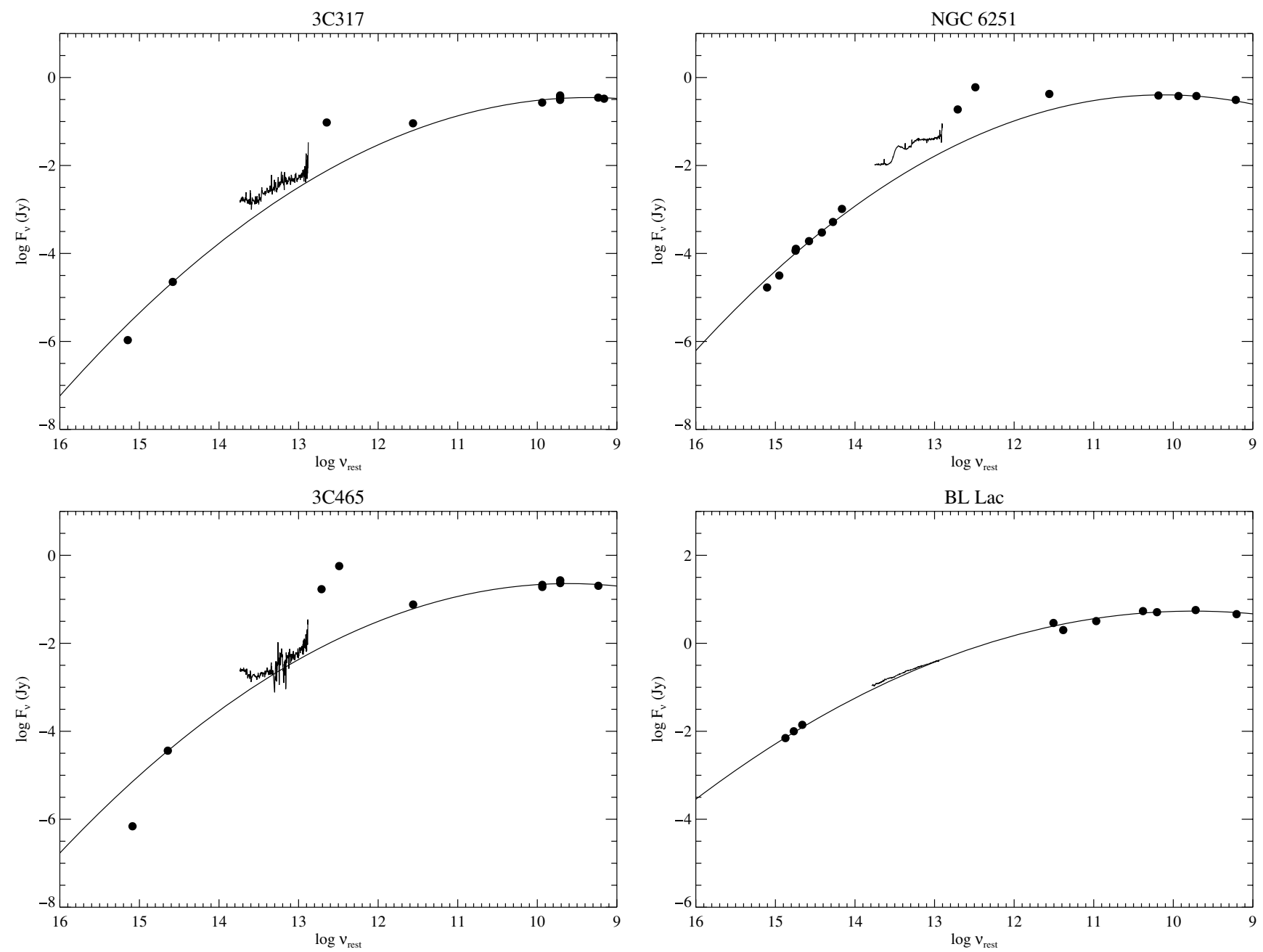

Figure 7. (Continued)

showed a "radically different" nuclear behavior (i.e., the FWHM of the nuclear source is much larger than for other CCCs and it is spatially resolved). This leaves 12 sources. In following works CCCs were also detected in 3C15 (R. Baldi 2009, private communication; as well as $\mathrm{M}$. Chiaberge 2009, private communication), in 3C189 (Capetti et al. 2002), in IC4296 (Balmaverde \& Capetti 2006a), and in NGC 6251 (Chiaberge et al. 2003). Thus, 16 sources here have an optical CCC detected (Table 1). Compared to other CCC objects, 3C386 stands out in the properties of its optical CCC which is exceptionally bright compared to the radio core. It has been suggested (Chiaberge et al. 1999), supported by the tentative detection of a broad optical emission line (Simpson et al. 1996), that this source shows additional optical flux from a BBB component, i.e., showing the central core of a type-1 AGN. However, it seems more likely that the foreground star which falls right on top of the nucleus (Lynds 1971; Madrid et al. 2006; Buttiglione et al. 2009) mimics an optical CCC because we do not see any signs for such a type- 1 AGN in the MIR. In fact, after subtracting some emission due to star formation the residual spectrum can be explained well by a quiescent early-type galaxy (Figure 13). In addition, new optical spectroscopy does not confirm a broad $\mathrm{H} \alpha$ emission-line component (Buttiglione et al. 2009). Because we cannot tell whether or not 3C386 itself shows a compact optical core we here exclude this object from further analysis. This leaves 15 sources where optical CCCs are securely detected.
Eleven of the 15 objects also show residual MIR emission, ${ }^{8}$ while the spectra of four sources are dominated by processes from the host galaxy (stellar emission, star formation).

We used the parabolic function to fit only the radio core data and the optical CCCs which are both claimed to be synchrotron emission from the same source (e.g., Chiaberge et al. 1999; Hardcastle \& Worrall 2000). For these data, we know that they are nuclear and fairly robust while other measurements might still be affected by either intrinsic absorption (UV) or aperture effects (MIR, FIR, submillimeter). ${ }^{9}$

\subsubsection{Sources with Residual MIR Emission}

In the following, we will discuss the SEDs on an object-toobject basis. References to flux measurements used for the SED plots (Figure 7) in addition to those already given in Table 1 are also noted. In Tables 2 and 3, we give the percentage contributions of the estimated nonthermal component to the observed MIR flux.

\footnotetext{
8 Recall that if our methodology is correct the presence of residual emission means they contain AGN-heated dust and/or prominent synchrotron contributions, i.e., a nuclear source of MIR continuum emission.

9 Because the core flux data were compiled from the literature they are generally not obtained simultaneously which can result in variability becoming important for the quality of the fits. However, in most cases all the multi-epoch data can be fitted well and any scatter present due to intrinsic variability seems relatively small.
} 
Table 2

Estimated Contributions to the MIR Spectra for Sources without Detected AGN Dust Components

\begin{tabular}{|c|c|c|c|c|c|c|c|c|c|}
\hline \multirow[t]{2}{*}{ Object } & \multicolumn{4}{|c|}{$F_{15 \mu \mathrm{m}}($ Rest Frame) } & \multicolumn{4}{|c|}{$F_{30 \mu \mathrm{m}}($ Rest Frame) } & \multirow[t]{2}{*}{ Comment } \\
\hline & $\begin{array}{l}\text { Total }^{\mathrm{a}} \\
(\mathrm{mJy})\end{array}$ & $\begin{array}{l}\text { SF } \\
(\%)\end{array}$ & $\begin{array}{c}\text { Stars } \\
(\%)\end{array}$ & $\begin{array}{c}\text { Synch }^{b} \\
(\%)\end{array}$ & $\begin{array}{l}\text { Total }^{\mathrm{a}} \\
(\mathrm{mJy})\end{array}$ & $\begin{array}{l}\mathrm{SF} \\
(\%)\end{array}$ & $\begin{array}{c}\text { Stars } \\
(\%)\end{array}$ & $\begin{array}{c}\text { Synch }^{\mathrm{b}} \\
(\%)\end{array}$ & \\
\hline \multicolumn{10}{|c|}{ Sources without optical core measurements } \\
\hline 3C76.1 & 1.72 & 21 & 0 & $\ldots$ & 3.53 & 30 & 0 & $\ldots$ & Low $\mathrm{S} / \mathrm{N}$ spectrum \\
\hline $3 \mathrm{C} 129$ & 3.26 & 17 & 46 & $\ldots$ & 11.44 & 14 & 6 & $\ldots$ & \\
\hline $3 \mathrm{C} 218$ & 4.49 & 100 & 0 & $\ldots$ & 15.91 & 100 & 0 & $\ldots$ & SF-dominated spectrum \\
\hline $3 C 293$ & 20.10 & 100 & 0 & $\ldots$ & 57.98 & 100 & 0 & $\ldots$ & SF-dominated spectrum \\
\hline $3 \mathrm{C} 386$ & 1.97 & 16 & 74 & $\ldots$ & 1.78 & 52 & 37 & $\cdots$ & Foreground star mimics CCC \\
\hline 3C403.1 & $<1.34$ & $\ldots$ & $\ldots$ & $\ldots$ & $<1.53$ & $\ldots$ & $\ldots$ & $\ldots$ & Not detected \\
\hline $3 \mathrm{C} 424$ & 1.52 & $\ldots$ & 12 & $\ldots$ & 5.06 & $\ldots$ & 2 & $\ldots$ & \\
\hline \multicolumn{10}{|c|}{ Sources with optical core measurements } \\
\hline $3 \mathrm{C} 29$ & 2.48 & $\ldots$ & 15 & 58 & 8.16 & $\ldots$ & 2 & 42 & Peak-up spillover ${ }^{\mathrm{c}}$, UV absorption \\
\hline 3C31 & 13.99 & 87 & 0 & 13 & 29.30 & 86 & 0 & 14 & SF-dominated spectrum \\
\hline $3 \mathrm{C} 66 \mathrm{~B}$ & 4.76 & $\ldots$ & 21 & 79 & 8.72 & $\ldots$ & 5 & 85 & \\
\hline $3 \mathrm{C} 83.1$ & 4.08 & 18 & 64 & 12 & 5.37 & 40 & 22 & 22 & Low PAH ratio \\
\hline 3C189 & 4.80 & $\ldots$ & 20 & 71 & 8.73 & $\ldots$ & 5 & 79 & Cold dust \\
\hline $3 \mathrm{C} 264$ & 9.11 & 52 & 0 & 48 & 23.88 & 67 & 0 & 33 & SF-dominated spectrum \\
\hline $3 \mathrm{C} 272.1$ & 28.08 & 30 & 57 & 13 & 46.70 & 53 & 15 & 15 & Cold dust, low PAH ratio \\
\hline $3 \mathrm{C} 274$ & 43.25 & $\ldots$ & 7 & 93 & 81.38 & $\ldots$ & 2 & 98 & \\
\hline IC 4296 & 10.02 & 6 & 30 & 69 & 19.88 & 9 & 7 & 68 & Cold dust \\
\hline 3C317 & 2.90 & $\ldots$ & 16 & 50 & 5.86 & $\ldots$ & 3 & 57 & \\
\hline $3 \mathrm{C} 465$ & 3.08 & $\ldots$ & 19 & 68 & 7.47 & $\ldots$ & 3 & 59 & Cold dust \\
\hline BL Lac & 246.45 & $\ldots$ & 0 & 100 & 381.92 & $\ldots$ & 0 & 100 & \\
\hline
\end{tabular}

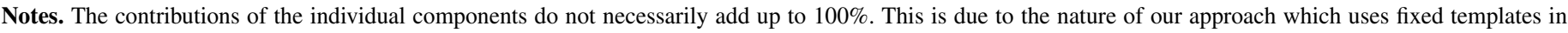

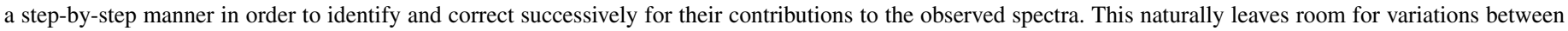

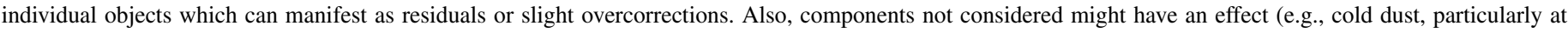

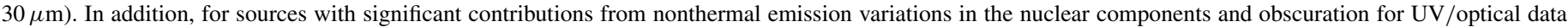
might result in some mismatch and residual emission. See the text for comments on individual sources where this might be the case.

${ }^{a}$ Flux measured from the observed MIR spectrum.

${ }^{\mathrm{b}}$ Flux of the synchrotron component shown in Figures 7 and 8.

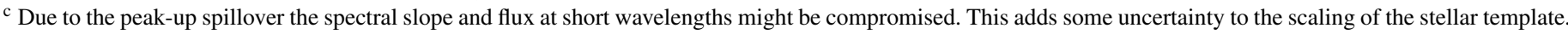

Table 3

Estimated Contributions to the MIR Spectra for Sources with Detected AGN Dust Components (see Notes to Table 2)

\begin{tabular}{|c|c|c|c|c|c|c|c|c|c|c|c|}
\hline \multirow[t]{2}{*}{ Object } & \multicolumn{5}{|c|}{$F_{15 \mu \mathrm{m}}$ (Rest Frame) } & \multicolumn{5}{|c|}{$F_{30 \mu \mathrm{m}}($ Rest Frame) } & \multirow[t]{2}{*}{ Comment } \\
\hline & $\begin{array}{l}\text { Total } \\
(\mathrm{mJy})\end{array}$ & $\begin{array}{l}\mathrm{SF} \\
(\%)\end{array}$ & $\begin{array}{c}\text { Stars } \\
(\%)\end{array}$ & $\begin{array}{c}\text { Synch } \\
(\%)\end{array}$ & $\begin{array}{c}\mathrm{AGN}^{\mathrm{a}} \\
(\%)\end{array}$ & $\begin{array}{l}\text { Total } \\
(\mathrm{mJy})\end{array}$ & $\begin{array}{l}\mathrm{SF} \\
(\%)\end{array}$ & $\begin{array}{c}\text { Stars } \\
(\%)\end{array}$ & $\begin{array}{c}\text { Synch } \\
(\%)\end{array}$ & $\begin{array}{c}\mathrm{AGN}^{\mathrm{a}} \\
(\%)\end{array}$ & \\
\hline $3 \mathrm{C} 84$ & 1307.88 & 1 & 0 & 12 & 86 & 3859.70 & 1 & 0 & 9 & 90 & \\
\hline $3 \mathrm{C} 120$ & 345.79 & 2 & 0 & $\ldots$ & 98 & 653.08 & 11 & 0 & $\ldots$ & 89 & Type-1 AGN \\
\hline $3 C 270$ & 27.45 & 7 & 21 & 11 & 61 & 52.14 & 27 & 5 & 16 & 52 & \\
\hline $\mathrm{E} 1821+643$ & 333.78 & 2 & 0 & $\ldots$ & 98 & 540.74 & 9 & 0 & $\ldots$ & 91 & Type-1 AGN \\
\hline
\end{tabular}

Notes.

a The contribution of the AGN component (which dominates in these objects) was determined as the total flux minus the other three contributions considered here.

b See comment on 3C29 in Table 2.

3C15. This source shows MIR continuum emission which is not due to either star formation or stellar emission (Figure 3). Using optical and NIR CCC measurements (provided by R. Baldi 2009, private communication; as well as M. Chiaberge 2009 , private communication) and radio core data, the nonthermal core component clearly underestimates the observed MIR flux.

However, we point out that $3 \mathrm{C} 15$ has a strong arcsecondscale jet (Leahy et al. 1997) which is also detected in the optical (Martel et al. 1998) and in X-rays (Kataoka et al. 2003). Since the extended jet is included in both IRS slits (SL slit oriented along the jet axis, LL slit oriented perpendicular to the jet axis) it can potentially make additional nonthermal contributions to the MIR spectrum. The inspection of the two-dimensional spectral images from IRS does not reveal any secondary component in the SL profile but the spectrum appears dominated by a single source (the size of the jet is almost comparable to the resolution of Spitzer at IRS wavelengths).

At $8.4 \mathrm{GHz}$, the total radio flux of the arcsecond jet is greater than the core flux by a factor of $\sim 10$ (Leahy et al. 1997; Hardcastle et al. 1998). Assuming a typical jet spectral index of $\alpha \sim-0.6$ (Bridle \& Perley 1984) this would lead to a 


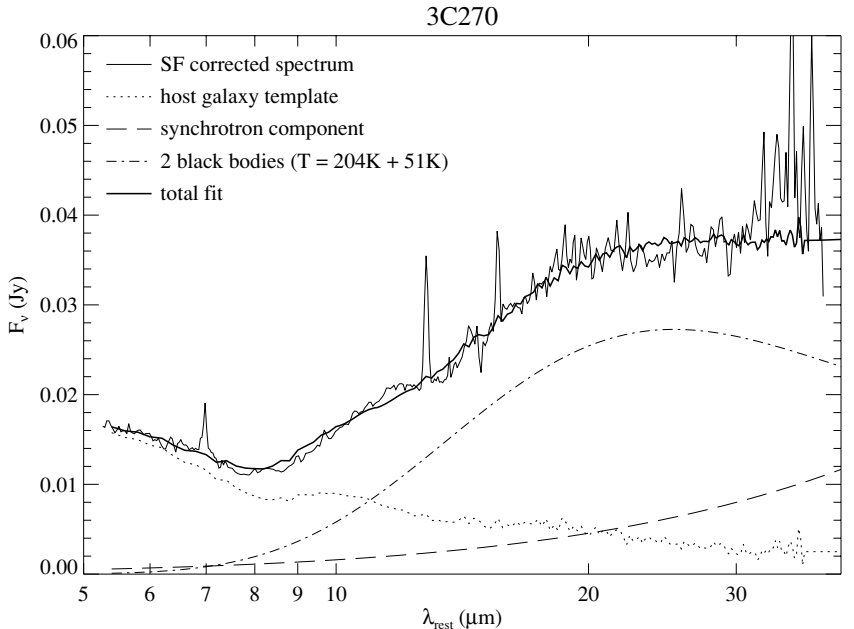

Figure 8. Star formation corrected spectrum of $3 \mathrm{C} 270$ is fitted with the spectrum of the elliptical galaxy NGC 1549 (dotted line), the synchrotron component from the core (dashed line), and two black bodies (dot-dashed line; see the text for details).

nonthermal jet contribution of $2 \mathrm{mJy}$ and $3 \mathrm{mJy}$ at $15 \mu \mathrm{m}$ and $30 \mu \mathrm{m}$, respectively, in the case of a single power law. For this conservative estimate we used a spectral index which is quite flat for a jet. In fact, the radio-optical spectral index of the X-ray
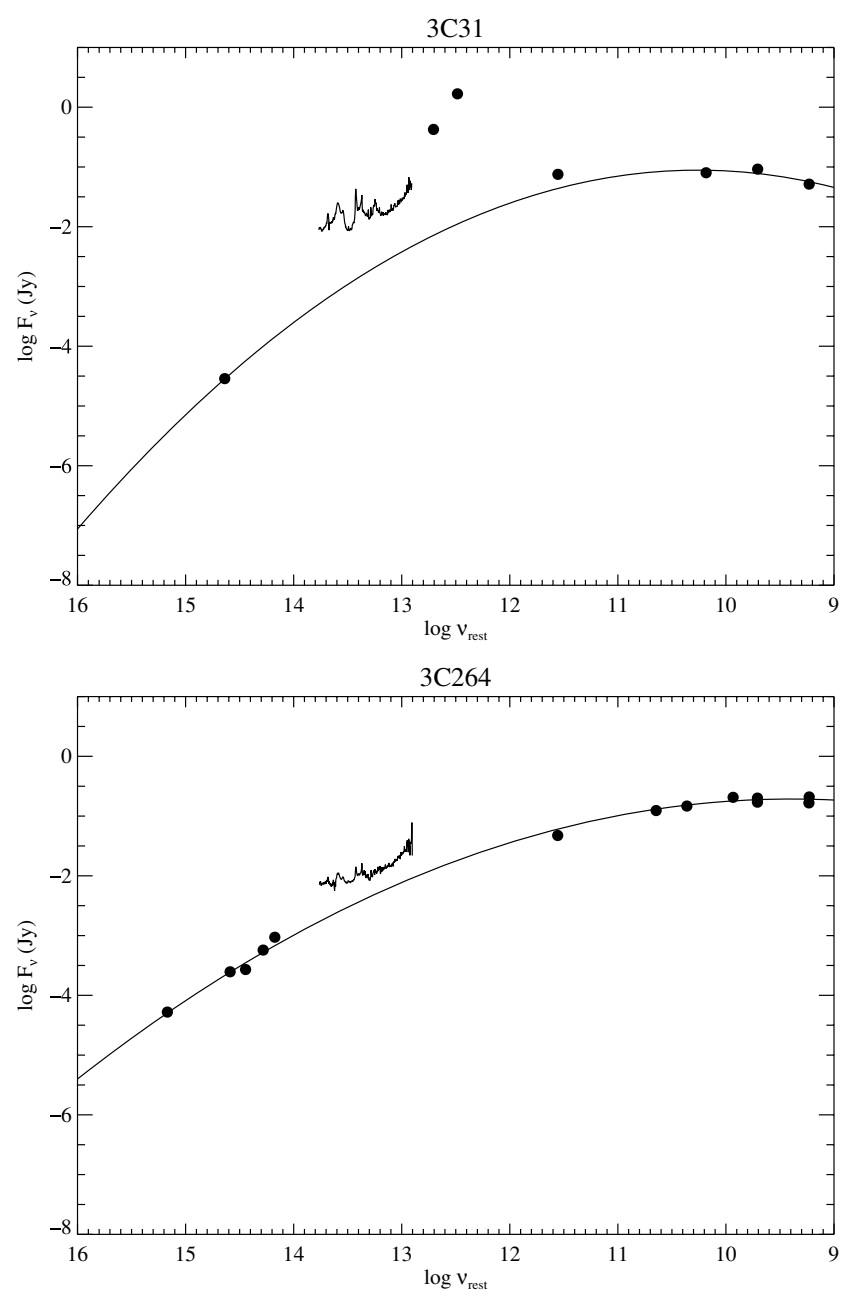

brightest jet knot ("Knot C"; Kataoka et al. 2003) is argued to be steeper than our chosen value (Kataoka et al. 2003; Dulwich et al. 2007).

Thus, even under the assumption of a conservatively flat spectral index for the jet the total nonthermal contribution (core + jet) to the MIR emission within the IRS apertures is still a factor of $\geqslant 2$ smaller than the total observed emission. In addition, the spectrum itself shows a change in slope around $\sim 20 \mu \mathrm{m}$ (which could be identified with a $18 \mu \mathrm{m}$ silicate feature) which argues for thermal contributions to the total flux.

Considering the appearance of the spectrum (including the potential $18 \mu \mathrm{m}$ silicate feature), the absence of notable PAH emission, and the difference between total flux and (conservatively estimated) nonthermal contributions we therefore argue that 3C15 shows thermal MIR emission from warm nuclear dust, possibly heated by an AGN.

We also note that Rinn et al. (2005) observe the X-ray core of $3 \mathrm{C} 15$ to be fairly weak $\left(L_{2-10 \mathrm{keV}} \sim 5 \times 10^{40} \mathrm{erg} \mathrm{s}^{-1}\right.$ intrinsic) but absorbed by a significant column density of $N_{\mathrm{H}} \sim 9 \times 10^{22} \mathrm{~cm}^{-2}$. References: Dicken et al. (2008), Hardcastle et al. (1998), and Morganti et al. (1997).

$3 C 29$. The parabolic fit matches the radio data quite well but greatly overpredicts the nuclear UV measurement. Assuming that this is due to extinction, dereddening of the UV flux in order to match it with the synchrotron fit would also result in increasing the optical flux somewhat. Such a synchrotron
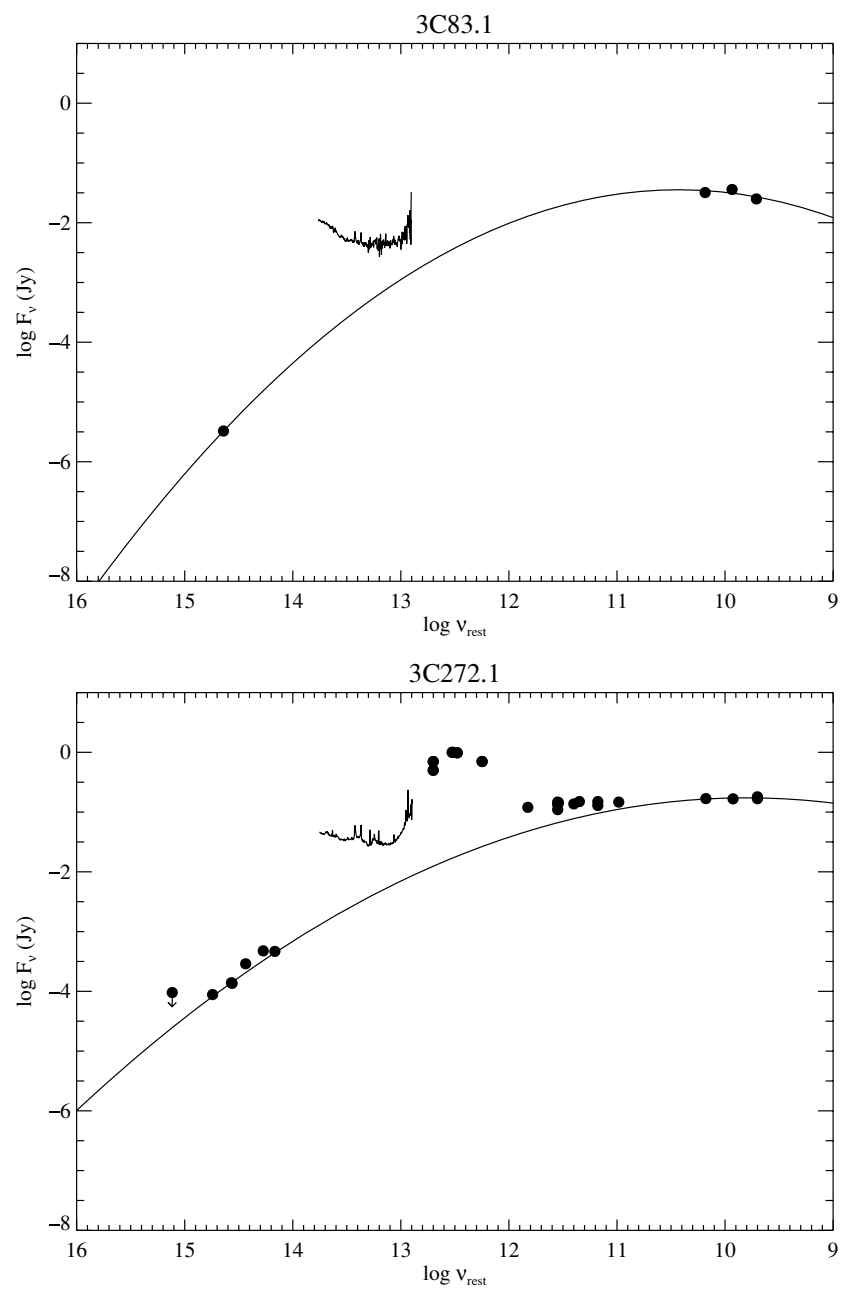

Figure 9. Same as in Figure 7 but for sources which are dominated by processes in the host galaxy (star formation and/or stellar emission). Here, we show the observed MIR spectra. 

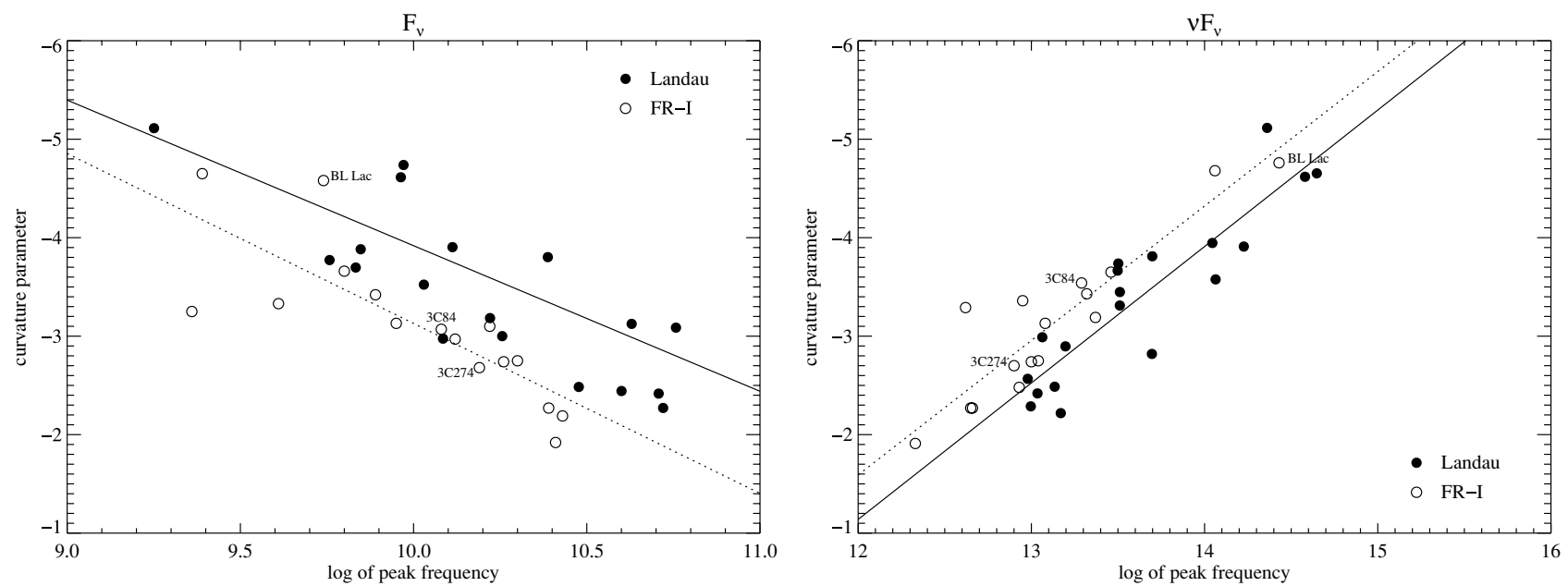

Figure 10. Curvature parameter plotted against the logarithm of the peak frequency for the core SED fits in $F_{v}$ (left) and $v F_{v}$ (right). Open symbols represent FR I sources, filled symbols the blazars from Landau et al. (1986). The solid and dashed lines show linear least-square fits to the blazar and FR I data points, respectively. Note that a smaller absolute number for the curvature parameter corresponds to a more strongly curved SED. We marked the sources BL Lac, 3C84, and 3C274 which are quite different from the lobe-dominated objects of our core sample but show extended radio emission of FR I type.

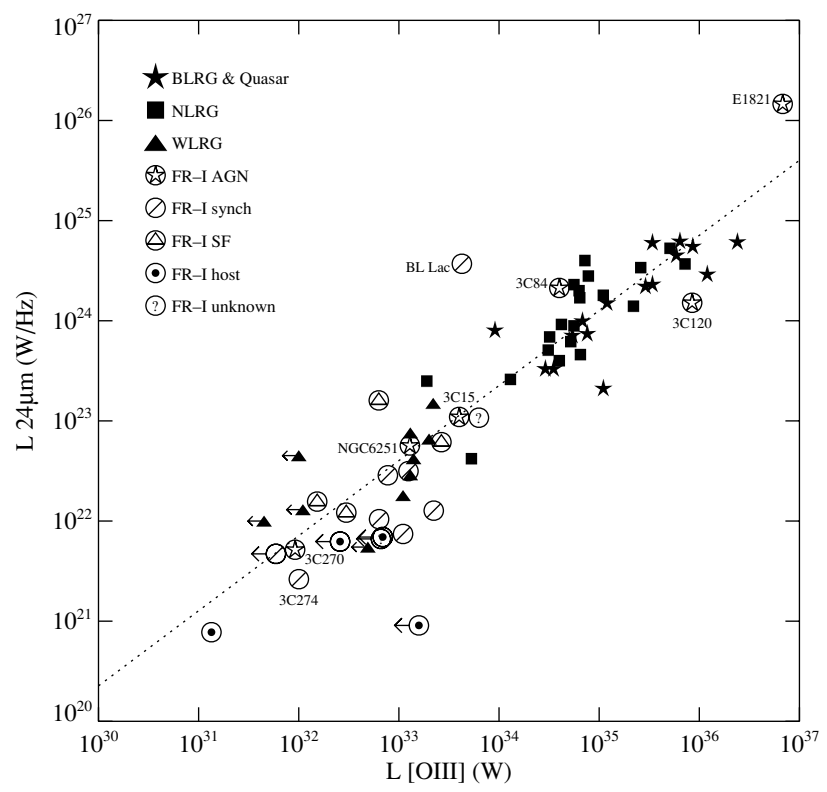

Figure 11. MIR luminosity at $24 \mu \mathrm{m}$ plotted vs. the [O III] emission-line luminosity. We show the objects presented in Dicken et al. (2009) as filled symbols and the FR Is from this paper as open symbols. The FR I points also indicate the dominating factor of the MIR emission: AGN, AGN-heated dust; synch, synchrotron emission; SF, star formation; host, stellar emission (plus possibly star formation). Sources labeled "unknown" (3C129 and 3C424) have MIR continuum emission in excess of host galaxy contributions but due to missing CCC measurements we cannot securely distinguish between AGN or synchrotron emission. The dashed line is the bisector of a linear least-square fit to the filled symbols (see the text for details).

SED could in principle account for the small excess in the MIR spectrum. In fact, Chiaberge et al. (2002) note that the UV point in 3C29 might be absorbed by a thin, extended dust lane. However, the mismatch of the UV measurement could also arise from variability. While the optical and UV data were obtained more than five years apart, the difference between the synchrotron estimate and the actual UV measurement corresponds to a factor of $\sim 30$ in flux. This seems high to be solely due to variability. Considering that some absorption in the UV/optical bands is present, that the slope of the MIR spectrum agrees reasonably well with the synchrotron fit, and that no host corrections were applied the MIR emission in this source is likely to be mostly nonthermal. References: Morganti et al. (1997) and Ricci et al. (2006).

$3 C 66 B$. Here, all data points (including the UV and MIR) agree very well with the parabolic fit. Thus, there does not seem to be significant extinction present in this object and the residual MIR emission is very likely to be nonthermal. At the shortest wavelengths of the MIR spectrum the stellar population of the host galaxy can be seen. 3C66B has an arcsecond-scale jet (Hardcastle et al. 1996) which is also seen in the optical (Butcher et al. 1980; Macchetto et al. 1991; Perlman et al. 2006), in X-rays (Hardcastle et al. 2001), and even at MIR wavelengths a detection with Infrared Space Observatory (ISO) is claimed (Tansley et al. 2000). However, unlike the case of $3 \mathrm{C} 15$, the jet in $3 \mathrm{C} 66 \mathrm{~B}$ is weaker than the core. Hardcastle et al. (2001) fit the radio through X-ray jet spectrum with a broken power-law model where the steepening from $\alpha \sim-0.5$ to $\alpha \sim-1.35$ occurs in the infrared. The radio-IR spectral slope agrees reasonably with the $14.5 \mu \mathrm{m}$ MIR jet flux of $\sim 1.7 \mathrm{mJy}$ estimated by Tansley et al. (2000). Considering these values the jet contribution in the MIR is only $<50 \%$ of that of the core. Thus, the inclusion of the jet in the IRS spectral aperture does not alter our conclusion for the dominance of nonthermal MIR emission in nucleus of 3C66B. References: Jackson et al. (1993), Xu et al. (2000), Quillen et al. (2003), and Kharb et al. (2005).

3C84. While this source is strongly core dominated in the radio, a clear dust emission bump is observed which reaches from the FIR well into the MIR. The SED is very complex and suffers from resolution effects (submillimeter versus Very Large Array (VLA) versus Very Long Baseline Array (VLBA)), which reduce the quality of the synchrotron core fit. In addition the VLBA data suggest self-absorption in the core making the SED asymmetric which cannot be fitted well with a parabolic function. Therefore, the fit was limited to the optical core and the radio core measurements excluding the $5 \mathrm{GHz}$ data where the downturn in the SED is already quite prominent. Besides the resolution effects and the self-absorbed core, variability might also be important and the fit cannot be more than a rough approximation of the synchrotron core component in this case. However, the thermal MIR and FIR bump is very pronounced in $3 \mathrm{C} 84$ and variations in the underlying synchrotron component are almost negligible at these wavelengths. 

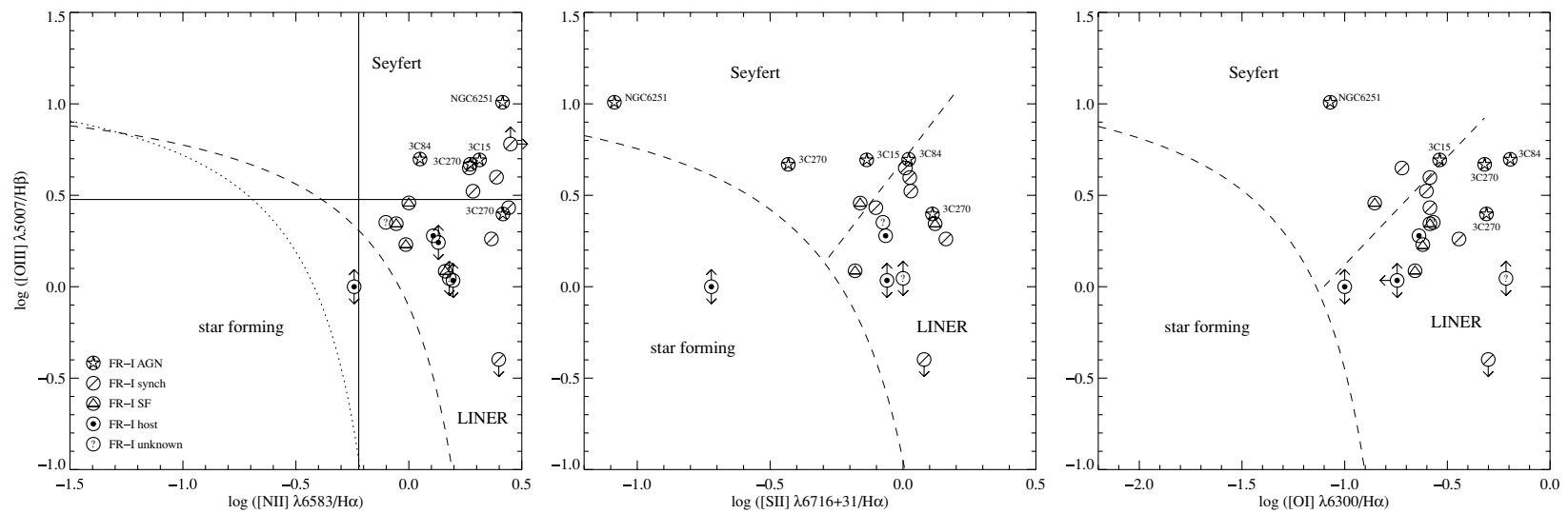

Figure 12. Diagnostic optical emission-line diagrams. On the left the dotted line is the dividing line used by Kauffmann et al. (2003) and the dashed line corresponds to the dividing line of Kewley et al. (2001). Sources with [N II] $/ \mathrm{H} \alpha>0.6$ are often defined as Seyferts for [O III]/H $\beta>3$ and as LINERs for [O III]/H $\beta<3$. For the diagrams in the middle and on the right the dividing lines from Kewley et al. (2006) are used. The symbols are the same as in Figure 11. For 3C270, the data point with the higher $[\mathrm{O} \mathrm{III}] / \mathrm{H} \beta$ ratio corresponds to the small aperture $H S T$ data while the lower flux ratio represents the ground-based results (see the text).

The MIR spectrum itself does not show significant contribution from stellar emission, only minor indications for star formation, and overall has a spectral appearance quite similar to "thermal" spectra of AGN powered sources. The fact that the spectrum is on average almost an order of magnitude brighter than the underlying nonthermal core emission strengthens the argument for thermal dust emission in 3C84, powered by a central engine. In addition, Ho et al. (1997b) find broad emission line components in $\mathrm{H} \alpha$ and other permitted lines. References: Knapp \& Patten (1991), Quillen et al. (2003), Haas et al. (2004), Stickel et al. (2004), and Taylor et al. (2006).

3C189. In the observed MIR spectrum this source is very similar to 3C66B (Figure 1). Also in the context of the SED we see that the fit to the synchrotron core matches with the MIR emission in shape as well as in flux. An IRAS $100 \mu \mathrm{m}$ flux measurement indicates the presence of cool (possibly extended) dust. References: Bridle \& Fomalont (1978), Knapp et al. (1990), and Giovannini et al. (1994).

3 C270. Including nuclear NIR measurements as well as (large aperture) FIR and (sub)millimeter data, the SED has very good coverage. We see that the radio core data, the NIR, and the (sub)millimeter data fitted well with the synchrotron prediction, arguing for their common nonthermal origin. The MIR spectrum, however, shows a very clear excess over the underlying nonthermal emission. Not only does the excess in MIR flux indicate additional thermal emission from warm dust in this source, but also the shape of the Spitzer spectrum makes its nature as nonthermal emission very unlikely. The pronounced change of slope around $\lambda \sim 20 \mu \mathrm{m}$ rest wavelength (Figure 5) strongly suggest thermal emission from warm dust to be present in this source.

In fact, after subtracting the star-forming template from the spectrum the MIR continuum can be well fitted as a combination of the underlying synchrotron contribution and the elliptical template for the host galaxy, plus a black body with a temperature of $\sim 200 \mathrm{~K}$ (Figure 8). ${ }^{10}$ This emission from warm

\footnotetext{
10 The fit to the star formation corrected spectrum has only three variable parameters: the scaling of the elliptical template as well as the temperature of the two black bodies. Because the synchrotron component was determined independently from radio/optical core measurements its flux was held fixed. We note that the colder black body is not well constrained but including it improves the fit for $\lambda>20 \mu \mathrm{m}$. However, the presence or absence of this second black body leaves the temperature of the warmer component virtually unchanged as it is strongly constrained by the continuum between 10 and $20 \mu \mathrm{m}$.
}

dust is not powered by star formation because this would result in accompanying PAHs. At $15 \mu \mathrm{m}$, the continuum emission of this residual thermal MIR component has a luminosity of $v L_{v, 15 \mu \mathrm{m}} \sim 4 \times 10^{41} \mathrm{erg} \mathrm{s}^{-1}$ (see also Table 3).

In the optical, Barth et al. (1999) report a broad $\mathrm{H} \alpha$ line in polarized light. This (tentative) detection further supports the presence of a classical hidden AGN. Moreover, a geometrically thick nuclear dust disk has been observed in silhouette (Jaffe et al. 1993). We also note that the optical emission lines in the nuclear region have flux ratios expected for Seyferts (Ferrarese et al. 1996; see Section 4.8).

Several studies are in broad agreement on the basic X-ray properties like the moderate intrinsic luminosity of $L_{2-10 \mathrm{keV}} \sim$ $1 \times 10^{41} \mathrm{erg} \mathrm{s}^{-1}$ and the moderate absorption on the order of $N_{\mathrm{H}} \sim 5 \times 10^{22} \mathrm{~cm}^{-2}$ (Chiaberge et al. 2003; Gliozzi et al. 2003; Sambruna et al. 2003; Rinn et al. 2005; Zezas et al. 2005). The origin of the observed X-ray emission is, however, controversially discussed: while Chiaberge et al. (2003) interpret the X-ray power-law component as due to the jet, Gliozzi et al. (2003) argue in favor of an accretion flow as the most likely source for the bulk of the X-ray emission. On the other hand, Zezas et al. (2005) state that both processes are able to explain the observed X-ray properties. A Fe $\mathrm{K} \alpha$ line is (marginally) detected with an EW of $\sim 230$ eV (Gliozzi et al. 2003; Sambruna et al. 2003; Rinn et al. 2005) which is consistent with the upper limits from other studies (e.g., Chiaberge et al. 2003; Zezas et al. 2005).

While the warm dust might represent the same nuclear absorbing material detected in X-rays, we note that due to the large Spitzer slit width our spectra include the prominent $\sim 300 \mathrm{pc}$ nuclear dust disk. In principle, the $200 \mathrm{~K}$ dust observed in the MIR could correspond to this dust disk. Because in situ star formation as a heating mechanism can be largely excluded (no PAHs) another source of sufficient energy needs to be present in the nucleus of $3 \mathrm{C} 270$. Radiation from a (possibly hidden) active nucleus is an obvious possibility. However, in the case of heating by the low- to medium-luminosity AGN in 3C270 simple energy budget arguments strongly suggest the $\sim 200 \mathrm{~K}$ dust to reside on much smaller scales than $\sim 300 \mathrm{pc}$. References: Jones \& Wehrle (1997), Capetti et al. (2000), Jones et al. (2000), Quillen et al. (2003), Haas et al. (2004), and Kharb et al. (2005).

3C274. Interestingly, the slope of the Spitzer spectrum appears flatter than what one would expect according to the synchrotron core fit. After accounting for host galaxy light using 
the elliptical template the MIR continuum alone can be well fitted with a single power law of $\alpha \sim-0.80$ for wavelengths shorter than $\sim 25 \mu \mathrm{m}$. At longer wavelengths the spectral slope steepens with respect to the power law which might be due to an additional dust component (Perlman et al. 2007). As discovered by Whysong \& Antonucci (2001) and later discussed by Perlman et al. (2001) and Whysong \& Antonucci (2004), an unresolved $\sim 0$ '.5 MIR core is present in 3C274. The MIR spectrum is discussed in detail by Perlman et al. (2007). References: Knapp \& Patten (1991), Xu et al. (2000), Nagar et al. (2001), Quillen et al. (2003), Haas et al. (2004), Lister \& Homan (2005), Perlman et al. (2001, 2007), Shi et al. (2007a), and Whysong \& Antonucci (2004).

IC4296. For IC4296 the synchrotron fit falls very close to the MIR spectrum, but only for a very narrow wavelength interval $(\lambda \sim 20-30 \mu \mathrm{m})$. In this source a star-forming template has been subtracted because PAHs are clearly detected and the spectrum seems to be dominated by stellar emission shortward of $\lambda \sim 10 \mu \mathrm{m}$ (Figure 14). Above $25 \mu \mathrm{m}$ a considerable steepening is observed in the spectrum suggesting the presence of a thermal bump in the SED due to cooler dust which is supported by FIR measurements. Despite the influence of processes in the host galaxy at some IRS wavelengths, this source has a very strong nonthermal contribution of greater than $>60 \%$ at $15 \mu \mathrm{m}$ and $30 \mu \mathrm{m}$ (Table 2). Although the synchrotron signature is weaker than for sources like 3C66B or 3C189 it manifests as clear residual MIR emission. Interestingly, Pellegrini et al. (2003) argue that the X-ray core in IC4296 $\left(L_{2-10 \mathrm{keV}} \sim 1.6 \times 10^{41} \mathrm{erg} \mathrm{s}^{-1}\right.$ intrinsic) originates from the jet and the $\mathrm{X}$-rays are observed to be only mildly absorbed $\left(N_{\mathrm{H}} \sim 1 \times 10^{22} \mathrm{~cm}^{-2}\right)$. References: Killeen et al. (1986), Knapp et al. (1989), Morganti et al. (1997), Venturi et al. (2000), Pellegrini et al. (2003), and Ricci et al. (2006).

3 C317. For this source the MIR spectrum falls very close to the synchrotron fit in flux. The synchrotron curve overestimates the nuclear UV measurement somewhat and a similar situation of absorption intrinsic to the source or variability as in $3 \mathrm{C} 29$ could be imagined. In fact, Chiaberge et al. (2002) reported a factor of $\sim 10$ in variability for the nuclear UV point source. Thus, the discrepancy of the CCC measurements (and the offset in MIR flux) is likely to be caused by variability. The similarity in spectral shape and the reasonable match in flux (combined with the absence of star formation tracers) strongly suggest the dominance of nonthermal emission in the MIR. Spitzer FIR measurements at $70 \mu \mathrm{m}$ suggest the presence of cold dust in this object. References: Zhao et al. (1993), Venturi et al. (2000), and Quillen et al. (2003, 2008).

NGC 6251. Using UV, optical, and NIR measurements (Chiaberge et al. 2003) and multifrequency radio core measurements (VLBI; Evans et al. 2005a), a well sampled nuclear SED can be constructed for NGC 6251. As for other sources, variability might be an issue for the nonthermal core measurements and in fact variability with a factor of $\sim 2$ over a few years has been reported for the nuclear (VLBI) radio component (Evans et al. 2005a). However, the radio measurements shown here were obtained simultaneously and the fit also agrees well with most of the multi-epoch HST measurements. For this source the MIR spectrum shows a clear excess over the synchrotron core fit, even after the correction for some minor star formation as traced by PAH emission of small EW (Figure 1). The small contribution from star formation, the absence of signs for significant contributions from stellar emission, and the overall shape of the spectrum (including the strong silicate emission features) strongly suggests a considerable component of thermal MIR emission in the nuclear regions of NGC 6251. The spectrum also looks quite different from other sources which are dominated by nonthermal emission in the MIR (e.g., 3C66B, 3C189 in Figure 7).

At $30 \mu \mathrm{m}$, where the strong silicate emission features will have only a minor impact on the continuum luminosity (e.g., Spoon et al. 2007), we measure $v L_{v}=5.6 \times 10^{42} \mathrm{erg} \mathrm{s}^{-1}$ in the spectrum. On the other hand, the luminosity of the synchrotron component at this wavelengths is about $v L_{v} \sim 2.2 \times 10^{42} \mathrm{erg} \mathrm{s}^{-1}$, which is consistent with the $\nu L_{v} \sim 2.5 \times 10^{42} \mathrm{erg} \mathrm{s}^{-1}$ given by the synchrotron self-Compton SED fit of Chiaberge et al. (2003). We note, however, that the factor of $\sim 2.5$ luminosity difference between the synchrotron core and the MIR spectrum is of the order of the measured radio core variability. In principle, the excess of the spectrum could be accounted for by variability of the underlying synchrotron core component. Although this would change the thermal to nonthermal ratio in the MIR (with the nonthermal part potentially even dominating at times) the appearance of the MIR spectrum still argues for the existence of warm nuclear dust in NGC 6251.

Ferrarese \& Ford (1999) report the tentative detection of a broad component in $\mathrm{H} \alpha$ (this result seems to be strongly dependent on the assumed modeling of the narrow lines though). In X-rays the power-law component in NGC 6251 shows only very little absorption $\left(N_{\mathrm{H}} \sim 5 \times 10^{20} \mathrm{~cm}^{-2}\right)$ and has an intrinsic luminosity of $L_{2-10 \mathrm{keV}} \sim 4.8 \times 10^{42} \mathrm{erg} \mathrm{s}^{-1}$ (Gliozzi et al. 2004; Evans et al. 2005a). The additional detection of a broad $\mathrm{Fe} \mathrm{K} \alpha$ line (EW $\sim 220 \mathrm{eV}$ and FWHM $\sim 0.6 \mathrm{keV}$ ) led Gliozzi et al. (2004) to argue in favor of the presence of a standard accretion disk. In their study, the base of the jet is ruled out as the sole origin of the nuclear X-ray emission. Evans et al. (2005a) on the other hand question the significance of the $\mathrm{K} \alpha$ detection and favor the jet as the source for the bulk of the observed X-ray core emission. However, they do not exclude some contributions from an accretion flow. References: Golombek et al. (1988), Chiaberge et al. (2003), Quillen et al. (2003), and Evans et al. (2005a).

$3 C 465$. The observed spectrum falls very close to the synchrotron core fit. Assuming that the observed nuclear UV component is also nonthermal, significant absorption seems to be present in this source. Alternatively, variability of a factor of $\sim 10$ could cause this UV flux difference. As noted before, both processes can in principle account for the remaining offset between the MIR spectrum and the synchrotron fit. A steepening of the spectrum is observed for wavelengths larger than $\sim 30 \mu \mathrm{m}$ indicating a thermal bump at FIR wavelengths. This is supported by IRAS 60 and $100 \mu \mathrm{m}$ fluxes. References: Golombek et al. (1988), Venturi et al. (1995), Quillen et al. (2003), and Jetha et al. (2006).

$B L$ Lac. Using quasi-simultaneous flux measurements from Landau et al. (1983) we construct an SED of this object, which is here used as an example for a strongly aligned, highly beamed FR I source. Note that although no HST data were used the (ground-based) optical fluxes will be dominated by the core emission. The measurements can be well fitted by the parabolic function and even though the MIR spectrum was obtained at a different epoch, it fits extremely well with the estimate for the synchrotron core emission. The spectrum can be fitted with a power law with $\alpha \sim-0.7$. Reference: Landau et al. (1983).

\subsubsection{Sources without Residual MIR Emission}

In the following, we will present the sources with detected CCCs but where no significant residual MIR continuum 
emission can be identified. The observed spectra can be explained by star formation and/or stellar emission. The SED plots are shown in Figure 9.

$3 C 31$. This source is strongly dominated by star formation in the host galaxy (Figure 1). Its observed spectrum is almost an order of magnitude brighter than then underlying nonthermal component. Interestingly, the SL aperture for the MIR spectrum is roughly consistent with the size of the molecular disk observed in the center of this source (Okuda et al. 2005). IRAS $60 \mu \mathrm{m}$ and $100 \mu \mathrm{m}$ measurements indicate substantial amounts of colder FIR dust. References: Burch (1977), Xu et al. (2000), and Evans et al. (2005b).

$3 C 83.1$. The few existing data points for the core emission are well approximated by the parabolic fit. The total light spectrum is dominated by processes in the host galaxy and lies well above the synchrotron fit. The subtraction of the star-forming template leaves a negative residual at the location of the $7.7 \mu \mathrm{m}$ PAH feature (Figure 6). As discussed above, this cannot be accounted for by the stellar spectrum of the host galaxy and argues for a low $7.7 \mu \mathrm{m}$ to $11.3 \mu \mathrm{m}$ PAH ratio which might in fact be powered by low-luminosity AGN activity instead of star formation (see Section 4.1). References: O'Dea \& Owen (1986) and Xu et al. (1999).

$3 C 264$. While the observed spectrum of this source is dominated by star formation, it falls much closer to the synchrotron component than in 3C31. From Figure 9, we see that the nonthermal contribution might not be negligible (see also Table 2). However, without prior knowledge of this fact the source spectrum can be fitted by star formation only due to the wide variety in the MIR spectral properties of star-forming galaxies (e.g., Dale et al. 2005; Brandl et al. 2006; Smith et al. 2007).

We note that this source has an arcsecond-scale radio jet (e.g., Lara et al. 2004) which is also detected at optical wavelengths (Crane et al. 1993; Baum et al. 1997). However, in the radio the core is stronger than the total jet emission and has a significantly flatter spectrum (Lara et al. 2004). While the jet emission steepens even more at optical wavelengths the contributions in the MIR are negligible compared to the core. References: $\mathrm{Xu}$ et al. (2000), Lara et al. (2004), and Kharb et al. (2005).

$3 C 272.1$. The unusual sharp turn from a slightly blue slope into a steep, red slope observed in the MIR spectrum of this source is supported in the framework of the SED where several FIR measurements indicate the presence of a strong thermal bump from cool dust. The spectrum lies well above the core synchrotron fit at almost all wavelengths and comes closest near $\lambda \sim 25 \mu \mathrm{m}$ where the slope change takes place (Figure 6). The combination of strong PAH emission and a very low PAH $7.7 \mu \mathrm{m}-11.3 \mu \mathrm{m}$ ratio results in a prominent depression at the location of the $7.7 \mu \mathrm{m}$ feature after subtraction of the starforming template. As discussed above (Section 4.1) the unusual $\mathrm{PAH}$ ratio might point toward AGN excitation instead of star formation but we count this source here as host dominated. References: Bower et al. (2000), Leeuw et al. (2000, 2004), Nagar et al. (2001), Haas et al. (2004), and Doi et al. (2005).

\subsection{Sources without CCC Measurements}

In Section 4.4, we study the contributions of the synchrotron cores to the MIR emission in our sources. However, this test is limited to objects where optical CCC measurements are available. When using the CCC fluxes in combination with radio core data, a nuclear SED can be constructed which bridges the MIR wavelengths. This way a fairly robust estimate of the nonthermal component can be achieved which helps to distinguish between thermal and nonthermal contributions to any residual MIR emission (i.e., emission not resulting from star formation or stellar processes).

Where no CCC measurements are available, a reliable estimate of the synchrotron core contribution to the MIR emission is not possible. However, the overall shape of the spectrum might still provide indications on which process - thermal or nonthermal-dominates the MIR emission. Out of the 25 sources considered in this paper, seven objects do not have CCC measurements available. We here briefly comment on these seven sources.

3C76.1. The MIR spectrum of this source can be well explained by a combination of star formation and stellar emission. No significant residual continuum can be identified. However, this spectrum suffers from low $\mathrm{S} / \mathrm{N}$ which limits the reliability of any conclusions regarding the MIR continuum constituents.

3C129. After correcting for some contributions from star formation and accounting for stellar emission from the host galaxy, 3C129 shows strong, red continuum emission longward of $\sim 15 \mu \mathrm{m}$ (Figure 13). Inspecting Figure 13 reveals that we could in principle have used a star formation template with a steeper MIR/FIR slope which would reduce the level of residual continuum. However, given the lack of any strong optical emission lines, the general appearance of the optical spectrum, and the clear dominance of an old stellar population (as determined by Buttiglione et al. 2009), it seems unlikely to us that this source harbors the stronger star formation which could power the redder MIR/FIR colors of a different template. However, while residual nuclear continuum emission is observed in 3C129 we cannot discriminate between a thermal or nonthermal origin because we lack optical CCC measurements. But we note that the residual MIR spectrum shows some concave upward curvature (Figure 13) and is significantly different in appearance compared to objects like 3C66B or 3C189 in which the MIR continuum is dominated by nonthermal emission (Figures 1 and 7). This argues in support of the thermal origin of the residual emission.

3C218. The MIR spectrum of Hydra A can be well explained by star formation only, perhaps with some minor contributions from stellar emission at the shortest MIR wavelengths (Figure 2). Considering the EW of the PAH features the continuum at $\lambda>20 \mu \mathrm{m}$ is in agreement with what can be expected for normal star-forming galaxies. The presence of a very young stellar population is also seen in optical spectra (Melnick et al. 1997; Aretxaga et al. 2001; Wills et al. 2004).

3C293. The MIR spectrum of 3C293 is strongly dominated by star formation and no residual nuclear continuum can be identified (Figure 1). In these properties it is quite similar to $3 \mathrm{C} 31$.

3C424. For 3C424 no significant contributions from star formation can be identified in the MIR spectrum and a weak and noisy, but securely detected, continuum can be seen.

$3 C 120$ and E1821+640 are broad-line AGN, which have MIR spectra typical of their class, displaying strong silicate emission features and high ionization emission lines (Figure 2).

\subsection{Comparison with Blazars and Related Objects}

It has been suggested that most (but not all) FR I radio sources are the misaligned counterparts of BL Lac objects (Kollgaard et al. 1992; Urry \& Padovani 1995; Cassaro et al. 1999; Antonucci 2002a). As outlined in Section 4.4, the SEDs of these strongly beamed, highly core dominated sources are well described by the same parabolic function we utilized to fit 
the cores of the FR I objects (which is here used just as a fitting function for descriptive purposes).

In order to allow the comparison between the blazars and the FR I cores we used the quasi-simultaneous data from Landau et al. (1986) to fit the blazars in the same way as the FR Is. However, we limited the parabolic fit for the blazars to the radio ( $2 \mathrm{~cm}$ through $20 \mathrm{~cm}$ ) plus optical $R$-band measurements, thus maximizing the comparability with the FR I fits (the wavelengths of the nuclear FR I data used to constrain their fits were very similar). Overplotting all the blazar data onto the fits obtained using only the limited data points shows that this approach yields a reasonable representation of the curved SEDs.

We will now compare the resulting fit parameters for peak frequency and SED curvature of the (putatively) misaligned sources with the respective data of their highly aligned (supposed) siblings. Moreover, we performed the SED fits not only in $F_{v}$ but also in $v F_{v}$ using the same parabolic function as given in Equation (1) and using the same set of nuclear data.

As already shown in, e.g., Landau et al. (1986) a correlation of peak frequency and curvature in $F_{v}$ exists for blazars. For FR I sources a very similar behavior is detected (Figure 10). However, while both types of objects cover about the same range in $F_{v}$ peak frequencies there is a systematic offset for the FR Is toward more strongly curved SEDs. A comparable result has been obtained using broadband spectral indices of FR Is and lowenergy peaked BL Lacs. These two types of objects overlap in their radio/optical power-law slopes but there is a clear tendency for FR Is to have steeper $\alpha_{\text {ro }}$ than the BL Lacs (Hardcastle \& Worrall 2000; Trussoni et al. 2003; Balmaverde \& Capetti 2006a). Because for a parabola peaking at radio wavelengths (which is typically the case for the sources considered here) a steeper radio/optical single power-law slope corresponds to a higher curvature, our results shown in Figure 10 are consistent with these previous findings.

In $v F_{v}$ the blazars also show a correlation between peak frequency and curvature, but now the FR Is seem to continue this correlation at lower peak frequencies. However, we see no trend in the blazar distribution with FR morphology. ${ }^{11}$ In particular, blazars with extended radio luminosities typical for FR I objects do not necessarily concentrate toward the "classical" FR I objects in Figure 10, but seem to be randomly distributed within the blazar sample.

The lower peak frequency of FR Is in $\nu F_{v}$ could be understood as a result of different beaming factors for the emission dominating in blazars and FR I cores. This seems to be in agreement with the model of, e.g., Chiaberge et al. (2000b) who assume that the jet has a velocity structure with a fast moving central spine (which is strongly beamed and dominates in aligned sources) and a slower-but still relativistic_-outer layer of more isotropic, less strongly beamed emission which dominates in the misaligned cases. Due to a shift in peak frequency of the emission, different amounts of relativistic beaming for aligned (i.e., BL Lac) and nonaligned (i.e., FR I) sources would also affect the observed broadband spectral indices. This can serve as one possible explanation for the difference in $\alpha_{\text {ro }}$ (or SED curvature) between FR Is and BL Lacs (e.g., Chiaberge et al. 2000b; Trussoni et al. 2003; Balmaverde \& Capetti 2006a).

\footnotetext{
11 Using measurements for the extended radio luminosity associated with the blazars (Antonucci \& Ulvestad 1985), we introduce a luminosity threshold (and varying this threshold between $\log L_{1.4 \mathrm{GHz} \text {, ext }}$ of 31.5 and $32.5 \mathrm{erg} \mathrm{s}^{-1}$ $\mathrm{Hz}^{-1}$ ) for identifying "FR I blazars." Taking those blazars with extended radio luminosities below the threshold as the possible aligned counterparts for our misaligned FR I sources we see no trend of blazar FR type in Figure 14.
}

\subsection{MIR and Emission-line Luminosity}

Under the assumption that the narrow-line region of AGN is extended and not affected by nuclear obscuration it has been argued that the luminosity of the [O III] $\lambda 5007 \AA$ emission line can serve as an isotropic tracer of the intrinsic radiative AGN luminosity (Rawlings \& Saunders 1991; Tadhunter et al. 1998). It has also been shown that for luminous AGN the radiative power, as traced by [O III], is correlated with the MIR luminosity at $24 \mu \mathrm{m}$ (Tadhunter et al. 2007; Dicken et al. 2009). ${ }^{12}$

In Figure 11, we plot the luminosity at $24 \mu \mathrm{m}$ versus the luminosity in the [O III] emission line for the objects presented in Dicken et al. (2009, filled symbols) and for our FR I sources (open symbols). We also show as a dashed line the bisector of a linear least-square fit to the filled symbols. ${ }^{13}$ While the AGN FR Is on average fall close to the bisector the visual inspection of Figure 11 also reveals that the bulk of the remaining FR I sources tend to have lower $24 \mu \mathrm{m}$ luminosities. In fact, a Kolmogorov-Smirnoff (K-S) test of the displacement in $L_{24 \mu \mathrm{m}}$ from the fitted line gives only a $\sim 1 \%$ chance that two samples drawn from the same parent population would show a difference as strong as we see for the D09 sample and the non-AGN FR Is. ${ }^{14}$ The FR I AGN sources on the other hand are statistically consistent with being drawn from the same parent population as the D09 sample. We note that this comparison assumes that the $24 \mu \mathrm{m}$ flux is dominated by an AGN component. While this is a reasonable assumption for the majority of the sources in the D09 sample (which mainly consists of NLRGs and quasars) we have shown here that it is certainly not the case for the non-AGN FR Is. For the latter, the bulk of the MIR emission can be accounted for by other processes (Table 2).

The differences between FR Is with and without warm dust can also be established by comparing their respective MIR-to-[O III] luminosity ratios: we find $\log \left[v L_{v}(24 \mu \mathrm{m}) /\right.$ $L([\mathrm{O}$ III $])]=2.74 \pm 0.06$ for the four AGN FR Is but only a ratio of $2.06 \pm 0.20$ for FR Is without clear signs for warm dust emission. ${ }^{15}$ The results of this exercise are just as we would expect from the spectral analysis and provide a strong consistency check for our method.

\subsection{Diagnostic Diagrams}

Optical emission-line diagnostics are commonly used to identify the dominant activity type in line-emitting sources (e.g., Baldwin et al. 1981; Veilleux \& Osterbrock 1987; Kewley et al. 2001; Kauffmann et al. 2003). Although we here largely focus on the MIR continuum properties of our FR I sources, a brief study of their optical emission-lines ratios with respect to their

\footnotetext{
12 For distant FR II radio galaxies and quasars an interesting thread in the literature offers good evidence for the anisotropy of [O III], which we do not pursue here since we concentrate on FR I objects. But these interesting references are, e.g., Jackson \& Browne (1990), Hes et al. (1993), di Serego Alighieri et al. (1997), and Simpson (1998). See also Haas et al. (2005) for an infrared/optical comparison.

13 The bisector was determined using only the filled symbols and following the same strategy as Dicken et al. (2009): sources with upper limits on their [O III] emission were excluded as well as sources with clear starburst signatures in their optical spectra. For the rest of this section, we refer to this sample of 33 objects as the "D09 sample."

14 In order to match the selection criteria of those two samples we excluded FR I sources with [O III] upper limits from the K-S statistics as well as sources which are dominated by star formation (plus BL Lac).

15 We here exclude the luminous broad-line AGN 3C120 and E1821+643, the synchrotron-dominated sources BL Lac and 3C274 as well as the four sources which are dominated by emission from star formation (Table 2). Note also that the slope of the fitted line shown in Figure 11 is not equal one meaning that the $24 \mu \mathrm{m} /[\mathrm{O} \mathrm{III}]$ ratio changes with luminosity.
} 
Table 4

$24 \mu \mathrm{m}$ and [O III] Emission-Line Fluxes

\begin{tabular}{|c|c|c|c|}
\hline Object & $\begin{array}{c}F_{\text {rest }}(24 \mu \mathrm{m}) \\
(\mathrm{mJy})\end{array}$ & $\begin{array}{c}F([\mathrm{O} \mathrm{III}]) \\
\left(\mathrm{erg} \mathrm{s}^{-1} \mathrm{~cm}^{-2}\right)\end{array}$ & References \\
\hline $3 \mathrm{C} 15$ & 8.63 & $3.15 \times 10^{-15}$ & 1 \\
\hline $3 \mathrm{C} 29$ & 6.80 & $2.69 \times 10^{-15}$ & 1 \\
\hline $3 \mathrm{C} 31$ & 19.16 & $4.71 \times 10^{-15}$ & 1 \\
\hline $3 \mathrm{C} 66 \mathrm{~B}$ & 7.42 & $1.10 \times 10^{-14}$ & 1 \\
\hline $3 \mathrm{C} 76.1$ & 2.92 & $<2.91 \times 10^{-15}$ & 1 \\
\hline $3 \mathrm{C} 83.1$ & 4.46 & $<1.85 \times 10^{-15}$ & 1 \\
\hline $3 \mathrm{C} 84$ & 3130.13 & $5.89 \times 10^{-13}$ & 1 \\
\hline $3 \mathrm{C} 120$ & 618.30 & $3.47 \times 10^{-12}$ & 7 \\
\hline $3 \mathrm{C} 129$ & 6.95 & $<6.94 \times 10^{-15}$ & 1 \\
\hline $3 \mathrm{C} 189$ & 7.57 & $2.05 \times 10^{-15}$ & 5 \\
\hline $3 \mathrm{C} 218$ & 8.77 & $3.80 \times 10^{-15}$ & 8 \\
\hline $3 \mathrm{C} 264$ & 15.05 & $1.47 \times 10^{-15}$ & 1 \\
\hline $3 \mathrm{C} 270$ & 42.59 & $7.57 \times 10^{-15}$ & 2 \\
\hline $3 \mathrm{C} 272.1$ & 29.52 & $5.11 \times 10^{-15}$ & 1 \\
\hline $3 \mathrm{C} 274$ & 63.16 & $2.41 \times 10^{-14}$ & 1 \\
\hline IC4296 & 13.83 & $<1.74 \times 10^{-15}$ & 7 \\
\hline $3 \mathrm{C} 293$ & 34.60 & $1.36 \times 10^{-15}$ & 1 \\
\hline $3 \mathrm{C} 317$ & 4.74 & $8.32 \times 10^{-15}$ & 1 \\
\hline NGC 6251 & 41.98 & $5.20 \times 10^{-15}$ & 6 \\
\hline $3 \mathrm{C} 386$ & 1.45 & $<2.53 \times 10^{-14}$ & 1 \\
\hline $3 \mathrm{C} 424$ & 2.60 & $1.52 \times 10^{-15}$ & 1 \\
\hline $3 \mathrm{C} 465$ & 5.12 & $3.11 \times 10^{-15}$ & 1 \\
\hline E1821+643 & 527.33 & $2.45 \times 10^{-13}$ & 3 \\
\hline BL Lac & 332.69 & $3.82 \times 10^{-15}$ & 4 \\
\hline
\end{tabular}

References. (1) Buttiglione et al. 2009; (2) Ho et al. 1997a; (3) Kolman et al. 1991; (4) Lawrence et al. 1996; (5) SDSS; (6) Shuder \& Osterbrock 1981; (7) Tadhunter et al. 1993; (8) Wills et al. 2004.

MIR spectral "type" seems promising. We take data for the emission-line ratios either from the references given in Table 4 or from Hansen et al. (1995, 3C218) and from Lewis et al. (2003, IC4296).

In Figure 12, we show three emission-line ratio diagrams for the FR I objects (minus the broad-line sources 3C120 and E1821+643 and excluding BL Lac). Virtually all FR Is, regardless of their MIR properties, fall into the LINER or Seyfert region. Even the star-forming sources occupy the LINER region rather than showing $\mathrm{H}$ II-type emission-line ratios (this is discussed in the context of ULIRGs in, e.g., Antonucci 2002a, $2002 b$ ). Only one source (3C386) occupies the transition region (Figure 12, left) or the $\mathrm{H}$ II region (Figure 12, middle).

For the FR Is with warm dust emission, 3C15 and NGC 6251 fall into the Seyfert region for all three diagrams, which historically requires the presence of an active nucleus in these objects. 3C84 and 3C270 on the other hand can be classified as Seyferts or as LINERs depending on which diagram is used (Figure 12). This, however, also shows that sources which are LINERs optically can have significant emission from warm dust in the MIR. Interestingly, both of these LINERs with warm dust have broad emission lines detected: 3C84 shows a broad $\mathrm{H} \alpha$ component (Ho et al. 1997b) while Barth et al. (1999) report a (tentative) broad line in polarized light for 3C270. This is consistent with the findings of Sturm et al. (2006) who show that type-1 LINERs on average have an additional warm dust component compared to type-2 LINERs. Therefore, we can speculate that LINERs with warm dust emission will also show broad emission lines.

Because nuclear emission-line ratios can be diluted by more extended emission from, e.g., star formation, especially for the weak and elusive sources considered here, we demonstrate the importance of aperture effects in the case of 3C270 which appears twice on each diagram: for every line-ratio combination shown (Figure 12), 3C270 is always classified as a LINER when using the emission-line fluxes measured in a $2^{\prime \prime} \times 4^{\prime \prime}$ aperture (Ho et al. 1997a). However, considering only the line ratios for an $H S T$ spectrum of the very nuclear $00^{\prime \prime} 09 \times 00^{\prime \prime} .09$ (Ferrarese et al. 1996) places 3C270 in the Seyfert region for two out of the three diagrams.

Figure 12 also reveals that some of the FR Is which are dominated by synchrotron emission in the MIR (and which lack clear signs for substantial warm dust) exhibit Seyfert-like emission-line ratios and many fall very close to the LINER/ Seyfert transition line.

\section{SUMMARY AND CONCLUSIONS}

The MIR spectra of 25 FR I radio galaxies are presented. Many of these sources show contributions from stellar emission at the shortest MIR wavelengths observed. The spectra generally turn over from a blue to a red continuum slope (in $F_{v}$ ) around $\sim 10 \mu \mathrm{m}$. Signs for star formation as traced by PAHs is detected in several objects and the relative contribution to the total spectra range from not detected (e.g., 3C66B, 3C317) to minor (e.g., 3C129, 3C270) to dominant (e.g., 3C31, 3C293).

Focusing on sources with detected optical compact cores, the nuclear SEDs for 15 objects are fitted with a parabolic function in order to estimate the synchrotron contribution to the MIR emission. For seven out of 15 objects (47\%), the nuclear MIR emission turned out to be dominated by nonthermal processes: apart from the stellar contributions at short wavelengths, the MIR spectra of these sources are consistent with the optical and radio core measurements. MIR continuum emission in excess over the synchrotron cores is detected in the eight out of 15 remaining sources. For four out of those eight objects the spectra are dominated by processes related to the host galaxy (star formation, stellar emission). The remaining four sources $(27 \%)-3 \mathrm{C} 15,3 \mathrm{C} 84,3 \mathrm{C} 270$, and NGC 6251-show clear signatures of thermal MIR emission in excess over the nonthermal core contributions. This excess cannot be explained by either stellar emission or star formation, which argues for the presence of thermal dust emission powered by an AGN. At least one of these objects has been (tentatively) confirmed to have a hidden type-1 AGN with spectropolarimetry.

Thus, for most of our sources in which the nuclear MIR component can be identified (and is not masked by host galaxy emission) this emission is likely to be of nonthermal origin. But some FR I sources do exist which show evidence for a hidden AGN, consistent with findings at other wavelengths.

Parabolic functions were fitted to the radio and optical core data in order to estimate the nuclear synchrotron component. These fits were performed in $F_{v}$ as well as in $v F_{v}$. Comparing the fit parameters for the FR Is with those for a set of blazars and related objects, it appears that in $F_{v}$ both types of objects show a similar relation between the peak frequency and the curvature of the SED. However, while FR Is cover about the same peak frequency range as the blazars used here for comparison, they show systematically stronger curvature in their SEDs. This reflects in the fit parameters for $\nu F_{\nu}$ where the FR Is show on average smaller peak frequencies and again stronger SED curvature. Different amounts of relativistic beaming in BL Lacs and FR Is could serve as a possible explanation for these results.

The comparison with a correlation between MIR and optical emission-line luminosity found for luminous AGN reveals that the AGN-dominated FR Is are consistent with that relation 

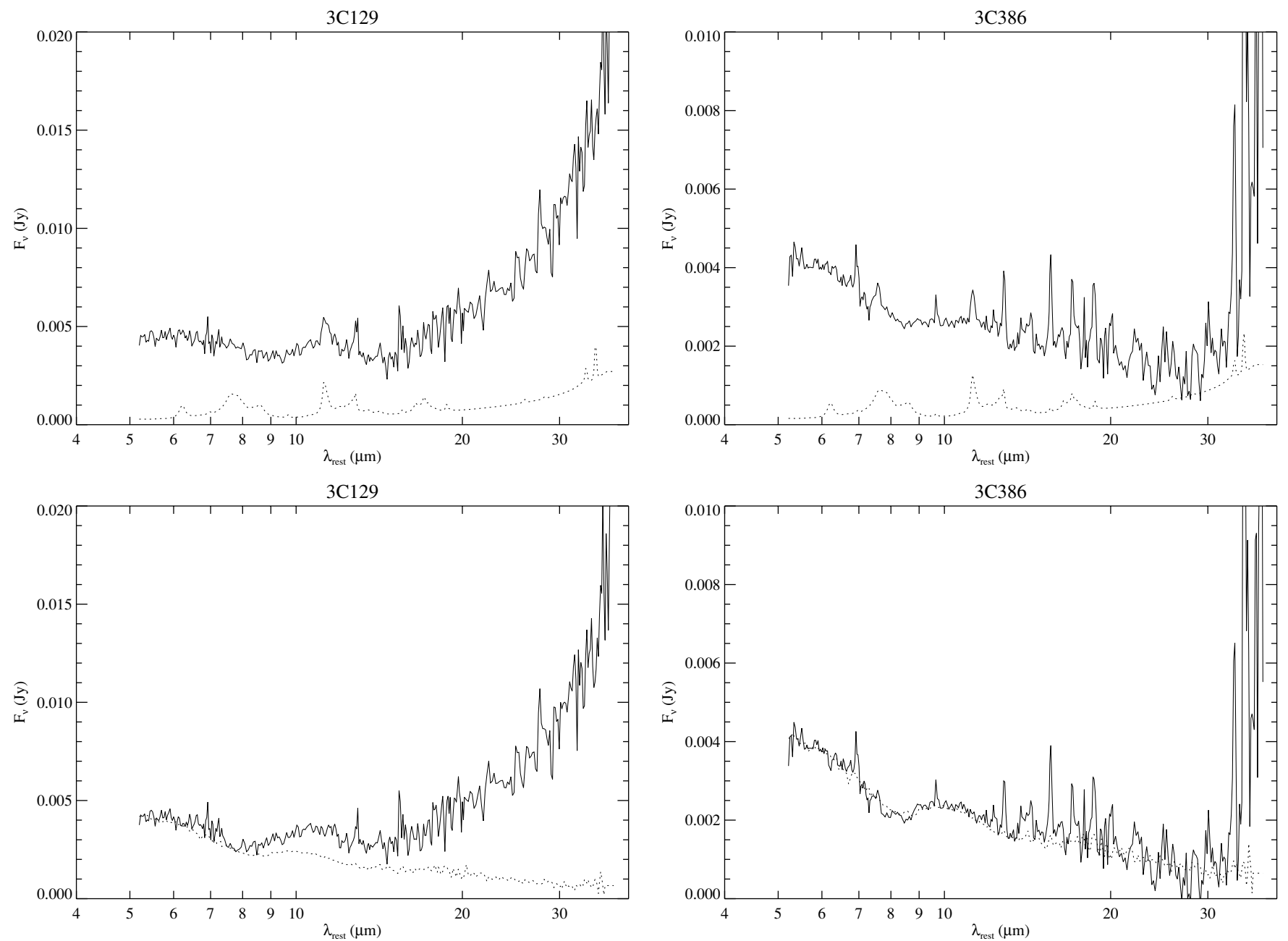

Figure 13. Subtraction of the star-forming template for $3 \mathrm{C} 129$ (left) and $3 \mathrm{C} 386$ (right). The top panel shows the observed spectrum with the scaled star-forming template. The bottom panel shows the corrected spectrum with a scaled spectrum of an early-type galaxy (NGC 1549).

while the non-AGN FR Is deviate substantially. In addition, we show that FR Is with and without warm dust differ significantly in their average $24 \mu \mathrm{m} /[\mathrm{O}$ III] luminosity ratio. The conclusion about the presence of an AGN in some of the FR Is is further strengthened by their optical emission-line ratios. These tests provide a strong consistency check for our method.

One important conclusion of this paper, as well as of other results in the literature, is that while most FR Is lack powerful type-1 AGN, it is not tenable to generalize on associations between FR I galaxies and "nonthermal only" AGN. A significant fraction of FR Is do have warm dust emission which could by attributed to hidden type-1 nuclei, a conclusion also anticipated on other grounds (e.g., Antonucci 2002a).

This work is based on observations made with the Spitzer Space Telescope, which is operated by the Jet Propulsion Laboratory, California Institute of Technology under a contract with NASA. Support for this work was provided by NASA through an award issued by JPL/Caltech. We have also made use of the NASA/IPAC Extragalactic Database (NED), which is operated by the Jet Propulsion Laboratory, California Institute of Technology, under contract with NASA. We thank Ann Wehrle for a critical reading of a previous version of this paper, and also Alan Marscher and Luis Ho for advice on specific issues. We are grateful to Marco Chiaberge for comments on a previous version of the paper and for newly mea- suring the optical compact core flux of $3 \mathrm{C} 15$. We are thankful for the critical comments of the referee which helped to improve the paper. This research has made use of Sloan Digital Sky Survey (SDSS) data products. Funding for the SDSS and SDSS-II has been provided by the Alfred P. Sloan Foundation, the Participating Institutions, the National Science Foundation, the U.S. Department of Energy, the National Aeronautics and Space Administration, the Japanese Monbukagakusho, the Max Planck Society, and the Higher Education Funding Council for England. The SDSS Web Site is http://www.sdss.org/.

Facility: Spitzer (IRS)

\section{APPENDIX \\ EXAMPLES FOR THE SUBTRACTION OF THE STAR-FORMING TEMPLATE}

Here, we present additional plots on the subtraction of the star formation component (Figures 13 and 14). The object 3C270 is already shown in Figures 5 and 9 while $3 \mathrm{C} 83.1$ as well as 3C272.1 can be found in Figure 6. This leaves 3C129, 3C386, and IC4296 as objects in which a significant correction due to emission from star formation was applied. We do not show objects which are clearly dominated by star formation in their MIR spectra (see Table 2). We also do not show objects where the contributions from star formation - as traced by the PAH features-is very minor and thus only a very small correction 

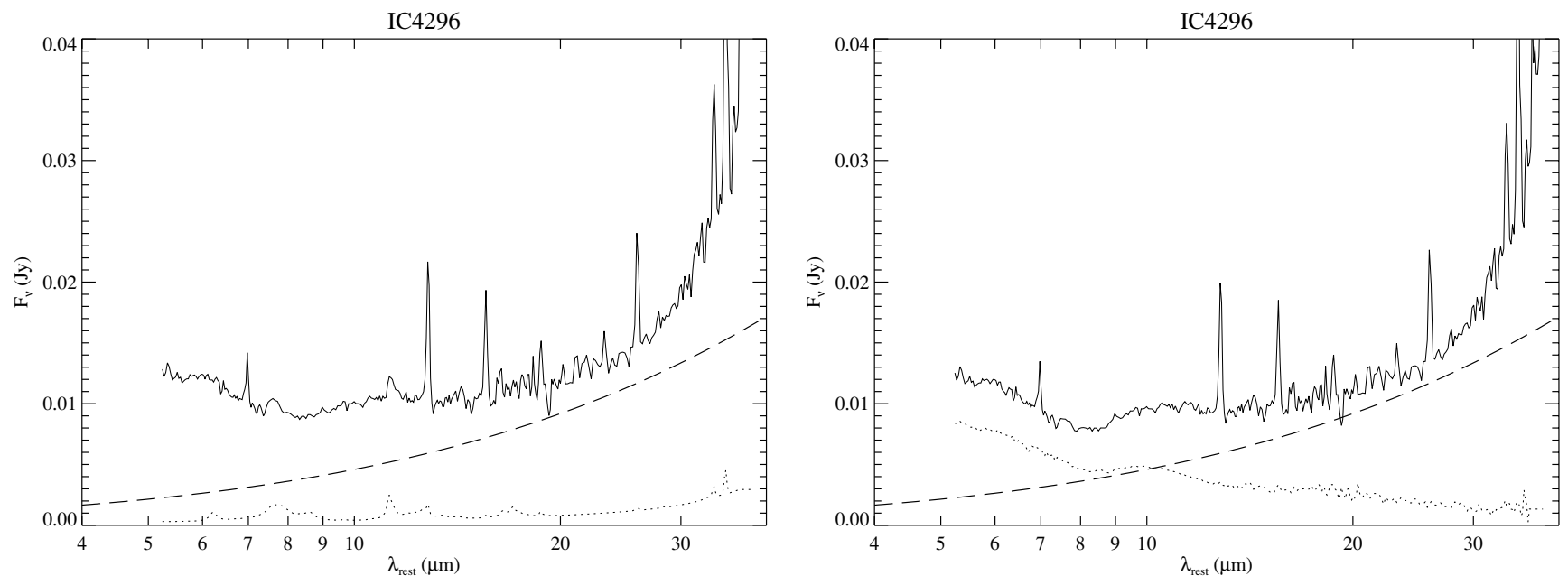

Figure 14. Subtraction of the star-forming template for IC4296. The panels are organized the same way as in Figure 13. Here, it becomes obvious what is mentioned in the note to Table 2: due to the strong relative contribution from the synchrotron core (long-dashed line; Figure 8) the scaling of the templates to features in the spectrum can result in overcorrections. Also, the steep slope at $\lambda>30 \mu \mathrm{m}$ hints at substantial emission from cold dust (which is supported by FIR measurements; Figure 7) might influence the measurement at $30 \mu \mathrm{m}$.

was made $(3 \mathrm{C} 84,3 \mathrm{C} 120$, NGC 6251, E1821+643; Tables 2 and 3).

\section{REFERENCES}

Antonucci, R. 1984, ApJ, 278, 499

Antonucci, R. R. J. 1986, ApJ, 304, 634

Antonucci, R. 1993, ARA\&A, 31, 473

Antonucci, R. 2002a, in Proc. of the XII Canary Islands Winter School of Astrophysics, Astrophysical Spectropolarimetry, ed. J. Trujillo-Bueno, F. Moreno-Insertis, \& F. Sánchez (Cambridge: Cambridge Univ. Press), 151

Antonucci, R. 2002b, in ASP Conf. Ser. 284, IAU Colloq. 184, AGN Surveys, ed. R. F. Green, E. Ye. Khachikian, \& D. B. Sanders (San Francisco, CA: ASP), 147

Antonucci, R. R. J., \& Ulvestad, J. S. 1985, ApJ, 294, 158

Aretxaga, I., Terlevich, E., Terlevich, R. J., Cotter, G., \& Díaz, Á. I. 2001, MNRAS, 325, 636

Baars, J. W. M., Genzel, R., Pauliny-Toth, I. I. K., \& Witzel, A. 1977, A\&A, 61,99

Baldwin, J. A., Phillips, M. M., \& Terlevich, R. 1981, PASP, 93, 5

Balmaverde, B., \& Capetti, A. 2006a, A\&A, 447, 97

Balmaverde, B., Capetti, A., \& Grandi, P. 2006b, A\&A, 451, 35

Barth, A. J., Filippenko, A. V., \& Moran, E. C. 1999, ApJ, 525, 673

Barthel, P. D. 1989, ApJ, 336, 606

Baum, S. A., et al. 1997, ApJ, 483, 178

Begelman, M. C., Blandford, R. D., \& Rees, M. J. 1984, Rev. Mod. Phys., 56, 255

Black, A. R. S., Baum, S. A., Leahy, J. P., Perley, R. A., Riley, J. M., \& Scheuer, P. A. G. 1992, MNRAS, 256, 186

Blundell, K. M., \& Rawlings, S. 2001, ApJ, 562, 5

Bower, G. A., et al. 2000, ApJ, 534, 189

Brandl, B. R., et al. 2006, ApJ, 653, 1129

Bressan, A., et al. 2006, ApJ, 639, 55

Bridle, A. H., \& Fomalont, E. B. 1978, AJ, 83, 704

Bridle, A. H., Fomalont, E. B., \& Cornwell, T. J. 1981, AJ, 86, 1294

Bridle, A. H., \& Perley, R. A. 1984, ARA\&A, 22, 319

Buchanan, C. L., Gallimore, J. F., O'Dea, C. P., Baum, S. A., Axon, D. J., Robinson, A., Elitzur, M., \& Elvis, M. 2006, AJ, 132, 401

Burch, S. F. 1977, MNRAS, 181, 599

Burns, J. O. 1990, AJ, 99, 14

Butcher, H. R., van Breugel, W., \& Miley, G. K. 1980, ApJ, 235, 749

Buttiglione, S., Capetti, A., Celotti, A., Axon, D. J., Chiaberge, M., Macchetto, F. D., \& Sparks, W. B. 2009, A\&A, 495, 1033

Cao, X., \& Rawlings, S. 2004, MNRAS, 349, 1419

Capetti, A., Axon, D. J., Chiaberge, M., Sparks, W. B., Macchetto, F. D., Cracraft, M., \& Celotti, A. 2007, A\&A, 471, 137

Capetti, A., Celotti, A., Chiaberge, M., de Ruiter, H. R., Fanti, R., Morganti, R., \& Parma, P. 2002, A\&A, 383, 104

Capetti, A., Morganti, R., Parma, P., \& Fanti, R. 1993, A\&AS, 99, 407
Capetti, A., Trussoni, E., Celotti, A., Feretti, L., \& Chiaberge, M. 2000, MNRAS, 318,493

Cassaro, P., Stanghellini, C., Bondi, M., Dallacasa, D., della Ceca, R., \& Zappalà, R. A. 1999, A\&AS, 139, 601

Chiaberge, M., Capetti, A., \& Celotti, A. 1999, A\&A, 300, 643

Chiaberge, M., Celotti, A., Capetti, A., \& Ghisellini, G. 2000b, A\&A, 358, 104

Chiaberge, M., Gilli, R., Capetti, A., \& Macchetto, F. D. 2003, ApJ, 597, 166

Chiaberge, M., Macchetto, F. D., Sparks, W. B., Capetti, A., \& Martle, A. 2002, ApJ, 571, 247

Cleary, K., Lawrence, C. R., Marshall, J. A., Hao, L., \& Meier, D. 2007, ApJ, 660, 117

Cohen, M. H., Ogle, P., Tran, H. D., Goodrich, R. W., \& Miller, J. S. 1999, AJ, 118, 1963

Condon, J. J., \& Broderick, J. J. 1988, AJ, 96, 30

Condon, J. J., Frayer, D. T., \& Broderick, J. J. 1991, AJ, 101, 362

Crane, P., et al. 1993, AJ, 106, 1371

Dale, D. A., et al. 2005, ApJ, 633, 857

Das, M., Vogel, S. N., Verdoes Kleijn, G. A., O'Dea, C. P., \& Baum, S. A. 2005, ApJ, 629, 757

De Robertis, M. M., \& Yee, H. K. C. 1990, AJ, 100, 84

Dicken, D., Tadhunter, C., Axon, D., Morganti, R., Inskip, K. J., Holt, J., Delgado, R. G., \& Groves, B. 2009, ApJ, 694, 268

Dicken, D., Tadhunter, C., Morganti, R., Buchanan, C., Oosterloo, T., \& Axon, D. 2008, ApJ, 678, 712

di Serego Alighieri, S., Cimatti, A., Fosbury, R. A. E., \& Hes, R. 1997, A\&A, 328,510

Doi, A., Kameno, S., Kohno, K., Nakanishi, K., \& Inoue, M. 2005, MNRAS, 363, 692

Dulwich, F., Worrall, D. M., Birkinshaw, M., Padgett, C. A., \& Perlman, E. S. 2007, MNRAS, 374, 1216

Edge, D. O., Shakeshaft, J. R., McAdam, W. B., Baldwin, J. E., \& Archer, S. 1959, MNRAS, 68, 37

Evans, D. A., Hardcastle, M. J., Croston, J. H., Worrall, D. M., \& Birkinshaw, M. 2005a, MNRAS, 359, 363

Evans, A. S., Mazzarella, J. M., Surace, J. A., Frayer, D. T., Iwasawa, K., \& Sanders, D. B. 2005b, ApJS, 159, 197

Evans, D. A., Worrall, D. M., Hardcastle, M. J., Kraft, R. P., \& Birkinshaw, M. 2006, ApJ, 642, 96

Fanaroff, B. L., \& Riley, J. M. 1974, MNRAS, 167, 31

Ferrarese, L., \& Ford, H. C. 1999, ApJ, 515, 583

Ferrarese, L., Ford, H. C., \& Jaffe, W. 1996, ApJ, 470, 444

Giovannini, G., Feretti, L., Gregorini, L., \& Parma, P. 1988, A\&A, 199, 73

Giovannini, G., Feretti, L., Venturi, T., Lara, L., Marcaide, J., Rioja, M., Spangler, S. R., \& Wehrle, A. E. 1994, ApJ, 435, 116

Giovannini, G., Taylor, G. B., Feretti, L., Cotton, W. D., Lara, L., \& Venturi, T. 2005, ApJ, 618, 635

Gliozzi, M., Sambruna, R. M., \& Brandt, W. N. 2003, A\&A, 408, 949

Gliozzi, M., Sambruna, R. M., Brandt, W. N., Mushotzky, R., \& Eracleous, M. 2004, A\&A, 413, 139

Golombek, D., Miley, G. K., \& Neugebauer, G. 1988, AJ, 95, 26

Govoni, F., Falomo, R., Fasano, G., \& Scarpa, R. 2000, A\&A, 353, 507 
Gower, A. C., \& Hutchings, J. B. 1984, AJ, 89, 1658

Haas, M., Siebenmorgen, R., Schulz, B., Krügel, E., \& Chini, R. 2005, A\&A, 442,39

Haas, M., et al. 2004, A\&A, 424, 531

Hansen, L., Jorgensen, H. E., \& Norgaard-Nielsen, H. U. 1995, A\&A, 297, 13

Hardcastle, M. J., Alexander, P., Pooley, G. G., \& Riley, J. M. 1996, MNRAS, 278,273

Hardcastle, M. J., Alexander, P., Pooley, G. G., \& Riley, J. M. 1998, MNRAS, 296, 445

Hardcastle, M. J., Birkinshaw, M., \& Worrall, D. M. 2001, MNRAS, 326, 1499

Hardcastle, M. J., \& Worrall, D. M. 2000, MNRAS, 314, 359

Hes, R., Barthel, P. D., \& Fosbury, R. A. E. 1993, Nature, 362, 326

Heywood, I., Blundell, K. M., \& Rawlings, S. 2007, MNRAS, 381, 1093

Hines, D. C., Eilek, J. A., \& Owen, F. N. 1989, ApJ, 347, 713

Ho, L. C., Filippenko, A. V., \& Sargent, W. L. W. 1997a, ApJS, 112, 315

Ho, L. C., Filippenko, A. V., Sargent, W. L. W., \& Peng, C. Y. 1997b, ApJS, 112,391

Houck, J. R., et al. 2004, ApJS, 154, 18

Jackson, N., \& Browne, I. W. A. 1990, Nature, 343, 43

Jackson, N., Sparks, W. B., Miley, G. K., \& Macchetto, F. 1993, A\&A, 269, 128

Jaffe, W., Ford, H. C., Ferrarese, L., van den Bosch, F., \& O'Connell, R. W. 1993, Nature, 364, 213

Jetha, N. N., Hardcastle, M. J., \& Sakelliou, I. 2006, MNRAS, 368, 609

Jones, D. L., \& Wehrle, A. E. 1997, ApJ, 484, 186

Jones, D. L., Wehrle, A. E., Meier, D. L., \& Piner, B. G. 2000, ApJ, 534, 165

Jones, D. L., et al. 1986, ApJ, 305, 684

Kaneda, H., Onaka, T., Kitayama, T., Okada, Y., \& Sakon, I. 2007, PASJ, 59, 107

Kaneda, H., Onaka, T., \& Sakon, I. 2005, ApJ, 632, L83

Kaneda, H., Onaka, T., Sakon, I., Kitayama, T., Okada, Y., \& Suzuki, T. 2008, ApJ, 684, 270

Kataoka, J., Leahy, J. P., Edwards, P. G., Kino, M., Takahara, F., Serino, Y., Kawai, N., \& Martel, A. R. 2003, A\&A, 410, 833

Kauffmann, G., et al. 2003, MNRAS, 346, 1055

Kellermann, K. I., Pauliny-Toth, I. I. K., \& Williams, P. J. S. 1969, ApJ, 157, 1

Kewley, L. J., Dopita, M. A., Sutherland, R. S., Heisler, C. A., \& Trevena, J. 2001, ApJ, 556, 121

Kewley, L. J., Groves, B., Kauffmann, G., \& Heckman, T. 2006, MNRAS, 372 , 961

Kharb, P., Shastri, P., \& Gabuzda, D. C. 2005, ApJ, 632, L69

Killeen, N. E. B., Bicknell, G. V., \& Ekers, R. D. 1986, ApJ, 302, 306

Knapp, G. R., Bies, W. E., \& van Gorkom, J. H. 1990, AJ, 99, 476

Knapp, G. R., Guhathakurta, P., Kim, D.-W., \& Jura, M. A. 1989, ApJS, 70, 329

Knapp, G. R., \& Patten, B. M. 1991, AJ, 101, 1609

Kollatschny, W., Zetzl., M., \& Dietrich, M. 2006, A\&A, 454, 459

Kollgaard, R. I., Wardle, J. F. C., Roberts, D. H., \& Gabuzda, D. C. 1992, AJ, 104,1687

Kolman, M., Halpern, J. P., Shrader, C. R., \& Filippenko, A. V. 1991, ApJ, 373, 57

Kuehr, H., Witzel, A., Pauliny-Toth, I. I. K., \& Nauber, U. 1981, A\&AS, 45, 367

Lacy, M., Rawlings, S., \& Warner, P. J. 1992, MNRAS, 256, 404

Laing, R. A., \& Bridle, A. H. 1987, MNRAS, 228, 557

Laing, R. A., Bridle, A. H., Parma, P., Feretti, L., Giovannini, G., Murgia, M., \& Perley, R. A. 2008, MNRAS, 386, 657

Laing, R. A., \& Peacock, J. A. 1980, MNRAS, 190, 903

Laing, R. A., Riley, J. M., \& Longair, M. S. 1983, MNRAS, 204, 151

Lal, D. V., \& Rao, A. P. 2004, A\&A, 420, 491

Landau, R., Golisch, B., \& Jones, T. J., et al. 1986, ApJ, 308, 78

Landau, R., Jones, T. W., Epstein, E. E., Neugebauer, G., Soifer, B. T., Werner, M. W., Puschell, J. J., \& Balonek, T. J. 1983, ApJ, 268, 68

Lara, L., Giovannini, G., Cotton, W. D., Feretti, L., \& Venturi, T. 2004, A\&A, 415,905

Lawrence, C. R., Zucker, J. R., Readhead, A. C. S., Unwin, S. C., Pearson, T. J., \& Xu, W. 1996, ApJS, 107, 541

Leahy, J. P., Black, A. R. S., Dennett-Thorpe, J., Hardcastle, M. J., Komissarov, S., Perley, R. A., Riley, J. M., \& Scheuer, P. A. G. 1997, MNRAS, 291, 20

Leahy, J. P., \& Perley, R. A. 1991, AJ, 102, 537

Leeuw, L. L., Sansom, A. E., \& Robson, E. I. 2000, MNRAS, 311, 683

Leeuw, L. L., Sansom, A. E., Robson, E. I., Haas, M., \& Kuno, N. 2004, ApJ, 612,837

Lewis, K. T., Eracleous, M., \& Sambruna, R. M. 2003, ApJ, 593, 115

Lister, M. L., \& Homan, D. C. 2005, AJ, 130, 1389

Lynds, R. 1971, ApJ, 168, L87

Macchetto, F., et al. 1991, ApJ, 373, L55

Madrid, J. P., et al. 2006, ApJS, 164, 307

Martel, A. R., et al. 1998, ApJ, 496, 203
Meisenheimer, K., Haas, M., Müller, S. A. H., Chini, R., Klaas, U., \& Lemke, D. 2001, A\&A, 372, 719

Melnick, J., Gopal-Krishna,, \& Terlevich, R. 1997, A\&A, 318, 337

Miley, G. K., \& Osterbrock, D. E. 1979, PASP, 91, 257

Morganti, R., Killeen, N. E. B., \& Tadhunter, C. N. 1993, MNRAS, 263, 1023

Morganti, R., Oosterloo, T. A., Reynolds, J. E., Tadhunter, C. N., \& Migenes, V. 1997, MNRAS, 284, 541

Morganti, R., Oosterloo, T., Tadhunter, C. N., Aiudi, R., Jones, P., \& VillarMartin, M. 1999, A\&AS, 140, 355

Nagar, N. M., Wilson, A. S., \& Falcke, H. 2001, ApJ, 559, L87

Nieppola, E., Tornikoski, M., \& Valtaoja, E. 2006, A\&A, 445, 441

O’Dea, C. P., \& Owen, F. N. 1986, ApJ, 301, 841

Ogle, P., Antonucci, R., \& Whysong, D. 2007, in ASP Conf. Ser. 373, The Central Engine of Active Galactic Nuclei, ed. L. C. Ho \& J.-M. Wang (San Francisco, CA: ASP), 578

Ogle, P., Cohen, M. H., Miller, J. S., Tran, H. D., Fosbury, R. A. E., \& Goodrich, R. W. 1997, ApJ, 482, 37

Ogle, P., Whysong, D., \& Antonucci, R. 2006, ApJ, 647, 161

Okuda, T., Kohno, K., Iguchi, S., \& Nakanishi, K. 2005, ApJ, 620, 673

Owen, F. N., Ledlow, M. J., \& William, C. 1996, AJ, 111, 53

Pedlar, A., Ghataure, H. S., Davies, R. D., Harrison, B. A., Perley, R., Crane, P. C., \& Unger, S. W. 1990, MNRAS, 246, 477

Pellegrini, S., Venturi, T., Comastri, A., Fabbiano, G., Fiore, F., Vignali, C., Morganti, R., \& Trinchieri, G. 2003, ApJ, 585, 677

Perley, R. A., Bridle, A. H., \& Willis, A. G. 1984, ApJS, 54, 291

Perlman, E. S., Sparks, W. B., Radomski, J., Packham, C., Fisher, R. S., Piña, R., \& Biretta, J. A. 2001, ApJ, 561, 51

Perlman, E. S., et al. 2006, ApJ, 651, 735

Perlman, E. S., et al. 2007, ApJ, 663, 808

Quillen, A. C., Almog, J., \& Yukita, M. 2003, AJ, 126, 2677

Quillen, A. C., et al. 2008, ApJS, 176, 39

Rawlings, S., \& Saunders, R. 1991, Nature, 349, 138

Ricci, R., Prandoni, I., Gruppioni, C., Sault, R. J., \& de Zotti, G. 2006, A\&A, 445,465

Rinn, A. S., Sambruna, R. M., \& Gliozzi, M. 2005, ApJ, 621, 167

Sambruna, R. M., Gliozzi, M., Eracleous, M., Brandt, W. N., \& Mushotzky, R. 2003, ApJ, 586, L37

Shi, Y., Rieke, G. H., Hines, D. C., Gordon, K. D., \& Egami, E. 2007a, ApJ, 655,781

Shi, Y., et al. 2005, ApJ, 629, 88

Shi, Y., et al. 2007b, ApJ, 669, 841

Shuder, J. M., \& Osterbrock, D. E. 1981, ApJ, 250, 55

Simpson, C. 1998, MNRAS, 297, L39

Simpson, C., Ward, M., Clements, D. L., \& Rawlings, S. 1996, MNRAS, 281, 509

Smith, J. D. T., et al. 2007, ApJ, 656, 770

Spinrad, H., Marr, J., Aguilar, L., \& Djorgovski, S. 1985, PASP, 97, 932

Spoon, H. W. W., Marshall, J. A., Houck, J. R., Elitzur, M., Hao, L., Armus, L., Brandl, B. R., \& Charmandaris, V. 2007, ApJ, 654, 49

Stickel, M., Lemke, D., Klaas, U., Krause, O., \& Egner, S. 2004, A\&A, 422, 39

Strom, R. G., Willis, A. G., \& Wilson, A. S. 1978, A\&A, 68, 367

Sturm, E., et al. 2006, ApJ, 653, L13

Tadhunter, C. N., Morganti, R., di Serego-Alighieri, S., Fosbury, R. A. E., \& Danziger, I. J. 1993, MNRAS, 263, 999

Tadhunter, C. N., Morganti, R., Robinson, A., Dickson, R., Villar-Martin, M., \& Fosbury, R. A. E. 1998, MNRAS, 298, 1035

Tadhunter, C., Robinson, T. G., González Delgado, R. M., Wills, K., \& Morganti, R. 2005, MNRAS, 356, 480

Tadhunter, C., et al. 2007, ApJ, 661, 13

Tansley, D., Birkinshaw, M., Hardcastle, M. J., \& Worrall, D. M. 2000, MNRAS, 317,623

Taylor, G. B., Govoni, F., Allen, S. W., \& Fabian, A. C. 2001, MNRAS, 326, 2

Taylor, G. B., Gugliucci, N. E., Fabian, A. C., Sanders, J. S., Gentile, G., \& Allen, S. W. 2006, MNRAS, 368, 1500

Taylor, G. B., Perley, R. A., Inoue, M., Kato, T., Tabara, H., \& Aizu, K. 1990, ApJ, 360, 41

Temi, P., Brighenti, F., \& Matthews, W. G. 2007, ApJ, 660, 1215

Trussoni, E., Capetti, A., Celotti, A., Chiaberge, M., \& Feretti, L. 2003, A\&A, 403, 889

Urry, M., \& Padovani, P. 1995, PASP, 107, 803

Vallee, J. P. 1982, AJ, 87, 486

van Breugel, W., \& Jägers, W. 1982, A\&AS, 49, 529

Veilleux, S., \& Osterbrock, D. E. 1987, ApJS, 63, 295

Venturi, T., Castaldini, C., Cotton, W. D., Feretti, L., Giovannini, G., Lara, L., Marcaide, J. M., \& Wehrle, A. E. 1995, ApJ, 454, 735

Venturi, T., Morganti, R., Tzioumis, T., \& Reynolds, J. 2000, A\&A, 363, 84

Walker, R. C., Benson, J. M., \& Unwin, S. C. 1987, ApJ, 316, 546 
Weedman, D. W., et al. 2005, ApJ, 633, 706

Werner, M. W., et al. 2004, ApJS, 154, 1

Whysong, D., \& Antonucci, R. 2001, arXiv:astro-ph/0106381

Whysong, D., \& Antonucci, R. 2004, ApJ, 602, 116

Wills, K. A., Morganti, R., Tadhunter, C. N., Robinson, T. G., \& Villar-Martin, M. 2004, MNRAS, 347, 771

Wu, Q., Yuan, F., \& Cao, X. 2007, ApJ, 669, 96
Xu, C., Baum, S. A., O’Dea, C. P., Wrobel, J. M., \& Condon, J. J. 2000, AJ, 120,2950

Xu, C., O’Dea, C. P., \& Biretta, J. A. 1999, AJ, 117, 2626

Zezas, A., Birkinshaw, M., Worrall, D. M., Peters, A., \& Fabbiano, G. 2005, ApJ, 627,711

Zhao, J.-H., Sumi, D. M., Burns, J. O., \& Duric, N. 1993, ApJ, 416, 51

Zirbel, E. L., \& Baum, S. A. 2003, AJ, 125, 1795 